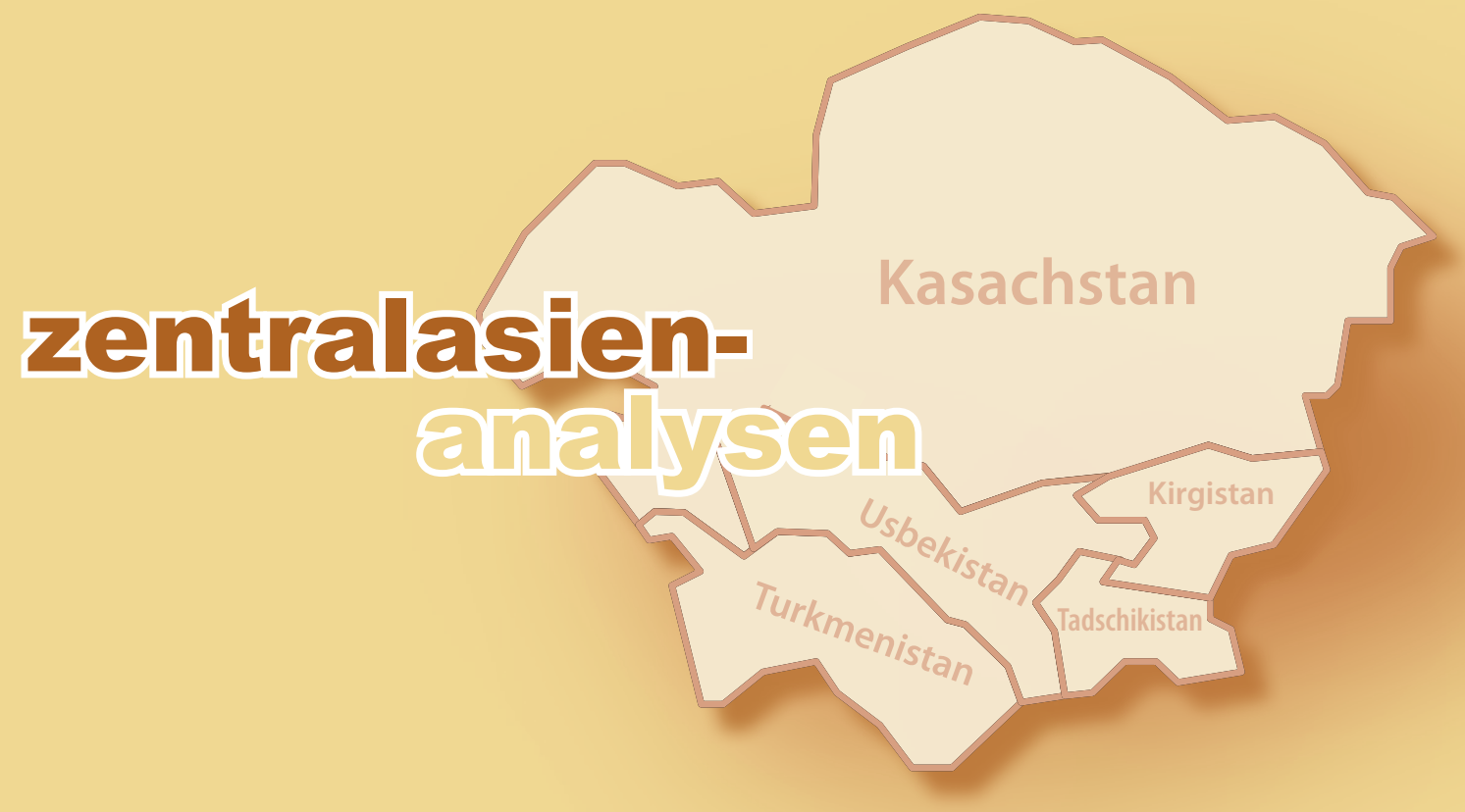

\title{
TERRORISMUSBEKÄMPFUNG VIA INTERNET IN ZENTRALASIEN
}

\section{ANALYSE}

Mangelnde Kreativität?

Terrorismusbekämpfung via Internet in Zentralasien

Von Anna Gussarova, Almaty

\section{TABELLEN UND GRAFIKEN ZUM TEXT}

Zahl der Google-Suchanfragen zum Islamischen Staat in Zentralasien

Meinungsumfragen in Kasachstan und Kirgistan zur Bekämpfung von Extremismus und Terrorismus

\section{CHRONIK}

20. Oktober - 23. November 2018

Kasachstan

Kirgistan

Tadschikistan

Turkmenistan

Usbekistan

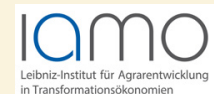

Leibniz-Institut für Ost- und Südosteuropaforschung

Zentrum für Osteuropa- und internationale Studien (ZOiS) $\mathrm{gGmbH}$ 


\section{Mangelnde Kreativität? Terrorismusbekämpfung via Internet in Zentralasien}

Von Anna Gussarova, Almaty

\section{Zusammenfassung}

Islamistische Extremisten nutzen immer mehr das Internet, um ihre Ideen zu propagieren, Anhänger zu rekrutieren und Pläne zu schmieden. Die Regierungen der zentralasiatischen Staaten verwenden bislang im Kampf gegen diese relativ neue Bedrohung ihrer Sicherheit fast ausschließlich repressive Methoden wie das Blockieren von Websites und die juristische Verfolgung von Internetnutzern, die extremistische Seiten liken oder weiterverbreiten. Beide Ansätze sind notwendig, haben aber in der Realität der zentralasiatischen Staaten Nachteile, wie begrenzte Effektivität und Einschränkungen der bürgerlichen Rechte. Die dritte Methode, Bildung und Aufklärung der Bevölkerung, wird dagegen nur selten genutzt, obwohl erste Ansätze erfolgversprechend sind. Insgesamt scheinen die Instrumente der Sicherheitskräfte der zentralasiatischen Staaten im Kampf gegen Extremismus in Internet altmodisch und wenig kreativ.

$\mathrm{D}_{\mathrm{p}}^{\mathrm{s}}$ Thema Extremismus ist in Zentralasien stark politisiert und wird vorrangig unter Sicherheitsaspekten betrachtet, es ist jedoch zugleich auch ziemlich unerforscht. Internetpropaganda für gewaltbereiten Extremismus, insbesondere in den sozialen Medien, ist für die Region ein relativ neues Phänomen. Erstmals tauchte sie 2014 in Form eines YouTube-Videos auf, das 150 kasachstanische Kämpfer zeigte, die sich dem Islamischen Staat (IS) angeschlossen hatten. Seitdem gehen von Zeit zu Zeit Nachrichten über aus Zentralasien stammende Terroristen, die Anschläge in Russland, Europa und auch in den USA verübt haben, um die Welt. Der tödliche Angriff, dem vor kurzem ausländische Fahrradtouristen in Tadschikistan zum Opfer fielen, zeigt, wie verwundbar die zentralasiatischen Gesellschaften in den letzten Jahren geworden sind und dass einige Menschen aus der Region für extremistische Propaganda, die zu Gewalt aufruft, empfänglich sind. In ihrem Kampf gegen den ihre Sicherheit bedrohenden, über das Internet verbreiteten gewaltbereiten Extremismus nutzen die Regierungen aller fünf zentralasiatischen Staaten repressive Methoden. Dabei werden drei Hauptinstrumente eingesetzt: technische, juristische und aufklärende Methoden.

\section{Filtern und Blockieren}

Bei den technischen Instrumenten geht es darum, extremistische und radikale Inhalte auf Internetseiten und in den sozialen Medien zu filtern oder zu blockieren. Seit 2013/14 haben $80 \%$ der extremistischen Inhalte im Internet mit Aktivitäten von Terrorgruppen im Irak und in Syrien zu tun, insbesondere von solchen des IS. Der Kampf gegen radikale Ideologien und extremistische Inhalte im Internet ist im Falle des IS, der sowohl englische wie auch russische Websites betreibt, besonders wichtig. Derzeit wird darüber spekuliert, dass sich der IS, nachdem er seine Bastionen in Syrien verloren hat, nach Afghanistan zurückzieht. Es wird interessant sein zu beobachten, ob sich seine Propaganda entsprechend ändert. Die Rekrutierung läuft - weltweit und insbesondere in Zentralasien - vor allem über dschihadistische Foren, in denen täglich Nachrichten, Mails und Meinungen ausgetauscht werden. Obwohl die Zahl der Accounts in den sozialen Medien zunimmt, sind Foren, in denen radikale Ideologien verbreitet werden, nach wie vor eines der bevorzugten Instrumente extremistischer Organisationen.

Früher wurde der Kampf gegen Terrorismus in Kasachstan mit dem Bemühen assoziiert, die Verteilung von gedrucktem Werbematerial und anderer religiöser Literatur zu unterdrücken. In den anderen zentralasiatischen Staaten, insbesondere in Tadschikistan, war dieser Ansatz ebenfalls sehr verbreitet. So wurden beispielsweise in Kasachstan im Jahre 2009 insgesamt 25.000 Exemplare solcher Druckerzeugnisse konfisziert; 2012 waren es nur noch 11.000. Man könnte also den Eindruck gewinnen, die Zahl extremistischer Materialien habe abgenommen. Man kann aber aus diesen Zahlen auch schließen, dass es in Zeiten des Internets und der massenhaften Digitalisierung nicht mehr nötig ist, Gedrucktes zu verbreiten. Aus diesem Grunde bemühen sich die zentralasiatischen Regierungen zurzeit darum, das Internet zu überwachen und radikale Inhalte zu identifizieren. Auf staatlicher Ebene sind vor allem die Sicherheitsapparate, einschließlich Strafverfolgungsbehörden, Nationale Sicherheitskomitees (die exklusiven Sicherheitsdienstleister in allen zentralasiatischen Staaten), Innenministerien, Büros der Generalstaatsanwälte sowie auch Ministerien für Information, Kommunikation und Kultur mit dieser Aufgabe befasst.

Nach Angaben des kirgisischen Dienstes von RFE/ $\mathrm{RL}$ gibt es 500 bis 600 Websites, die terroristische 
und extremistische Inhalte verbreiten und zum Dschihad, zum Widerstand gegen die Herrschenden und zur Errichtung eines Kalifats aufrufen. Es finden sich dort auch Anleitungen zum Bombenbau.

In Kasachstan wurden 2011 zum Beispiel 400 Internetseiten verboten, die radikale Ideen und Terrorismus propagierten. 2012 waren es bereits mehr als 500, und im Jahr 2013 mehr als 600. 2017 wurden nach Angaben des kasachstanischen Ministers für Information und Kommunikation, Dauren Abajew, mehr als 9.000 Internetseiten, die mit Terrorismus, Gewaltbereitschaft und Selbstmordattentaten zu tun hatten, gesperrt und mehr als 230.000 Beiträge gelöscht.

2016 haben das kirgisische Innenministerium und das Staatliche Komitee für nationale Sicherheit 86 Internetseiten wegen Propagierung von Terrorismus und Extremismus blockiert und verboten. Außerdem wurden über 20.000 Mail-Accounts von Odnoklassniki (ein russisches soziales Netzwerk, das in Zentralasien ebenso wie VKontakte sehr populär ist) als extremistisch eingestuft und gelöscht. 2017 wurde nach Angaben des Büros des kirgisischen Generalstaatsanwalts in Kirgistan der Zugang zu 159 Websites mit extremistischen und terroristischen Informationen blockiert. Dieselbe Situation können wir in Tadschikistan beobachten, wo die Regierung Facebook und Viber (den populärsten Nachrichtendienst im Land) sperren ließ, weil sie über den zunehmenden Einfluss radikaler Propaganda beunruhigt ist. In Usbekistan versucht man zurzeit, eine Liste von verbotenen Websites zu erstellen; sie wird jedoch auch viele weitere Quellen enthalten, die sich auf Pornographie, Extremismus, Terrorismus, Separatismus, Fundamentalismus, Aufstachelung zu nationalem, religiösem und ethnischem Hass, Zugang zu Drogen und Enthüllung von Staatsgeheimnissen beziehen. Es hat den Anschein, dass in Zentralasien viele Themen, die kontrovers diskutiert werden, politisiert und als Sicherheitsproblem eingestuft werden, insbesondere Religion.

Die technischen Werkzeuge haben eine zeitlich begrenzte Reichweite und ihre Effektivität ist daher ziemlich gering. Das simple Filtern und Blockieren von Websites führt dazu, dass Extremisten statt offener Kommunikations- und Informationskanäle geschlossene, wie Telegram, nutzen, wo sie schwieriger zu überwachen sind. Außerdem ist diese Vorgehensweise mit einer Reihe besonderer Schwierigkeiten verbunden. Erstens gibt es keine öffentlichen und verlässlichen Informationen von Seiten staatlicher Einrichtungen darüber, welche Internetquellen warum verboten oder blockiert sind. Die Definitionen von Extremismus und Terrorismus sind in allen zentralasiatischen Staaten vage und überschneiden sich. Zudem gehen die Justizbehörden selektiv vor bzw. die Rechtsstaatlichkeit ist schwach ausgeprägt. Daher können Hasstiraden und Widerspruch gegen staatliche Narrative im Internet ebenfalls unter den Extremismusvorwurf fallen. Aus diesem Grund ist es unmöglich, sich in den sozialen Medien sicher zu bewegen. Zweitens sind sich die Nutzer nicht bewusst und werden auch nicht darüber aufgeklärt, dass das Anschauen und Hören wie auch die Verbreitung im Internet zugänglicher extremistischer Materialien oder Quellen zu Gefängnisstrafen wegen Propagierung von Extremismus oder Terrorismus führen kann. Drittens zerstören die Regierungen der zentralasiatischen Staaten durch die Sperrung von sozialen Medien oder anderen Internet-Plattformen die ohnehin schwachen Identitätsbeziehungen und das Vertrauen innerhalb ihrer Zivilgesellschaften und verlieren zudem die Chance, die Lage an der Basis zu beobachten. Und schließlich sind die zentralasiatischen Staaten nicht in der Lage, Gegennarrative zur extremistischen Propaganda zu entwickeln und zu etablieren. Dieses Thema wird ausschließlich von internationalen Geldgebern und anderen Ländern behandelt, zum Beispiel von der OSZE, UNDP und UNODC sowie besonders von der EU und den Vereinigten Staaten.

Es liegt auf der Hand, dass Maßnahmen technischer Art, wie die Löschung gewalttätiger oder radikaler Inhalte, nachdrücklich umgesetzt werden sollten. Ein Beispiel ist die Entscheidung der EU, Google, Facebook und Twitter mit Strafen zu belegen, wenn sie extremistische Inhalte nicht innerhalb einer Stunde entfernen. Doch weitere, langfristige Problemlösungen sind als Vorbeugungsmaßnahmen gegen gewalttätigen Extremismus und radikale Ideologien ebenfalls wichtig.

\section{Gesetze kontra Freiheit des Internets}

Der zweite Weg, das Problem des Extremismus im Internet anzugehen, ist der der gesetzlichen Regelung. Nach Definition der vorhandenen, nicht sehr eindeutigen Gesetze zur »Bekämpfung des Extremismus« und »Bekämpfung des Terrorismus«, die in den zentralasiatischen Staaten in den 1990-er und frühen 2000-er Jahren verabschiedet wurden, handelt es sich bei extremistischen Materialien um jede Art von Information, die Hinweise auf und/oder direkte Aufrufe zu extremistischen Handlungen enthält oder ihre Notwendigkeit rechtfertigt. Zusätzlich enthalten die Strafgesetzbücher aller zentralasiatischen Staaten einen Artikel, der sich mit Aufstachelung zum Hass befasst (in Kasachstan ist damit Extremismus gemeint).

Die Gesetze enthalten zwar Definitionen des Extremismus und seiner Spielarten, aber sie sind sehr vage gehalten. Es ist nicht klar, ob der Begriff 'Aufstachelung sich auf Worte oder Handlungen oder beides bezieht. Die Bedeutung des Begriffs als solchem bleibt unklar. Davon abgesehen enthalten die Gesetze eine klare Bot- 
schaft: Aufrufe zur Gewalt werden als Extremismus eingeordnet, auch wenn darauf nicht unbedingt physische Gewaltakte folgen.

Um die innere Sicherheit zu gewährleisten ist es ist notwendig, die legitime Anwendung von staatlicher Gewalt zu stärken. Der gegenwärtig von den zentralasiatischen Regierungen verfolgte Ansatz zeigt aber auch eine starke Verschiebung der Balance von Bürgerrechten und Sicherheit zu Ungunsten der erstgenannten. In Zentralasien, wo bereits politische Rechte und bürgerliche Freiheiten, insbesondere die Meinungs-, Versammlungs- und Religionsfreiheit um der Stabilität willen eingeschränkt wurden, werden auch die sozialen Medien von den Regierungen manipuliert. Überall in der Region begann man - unter dem Vorwand der Extremismusbekämpfung - Menschen wegen der Verbreitung, der Weiterleitung und des Likens von "politisch, national oder religiös nicht korrekten « oder sensitiven Inhalten in den sozialen Medien zu verhaften. In Kasachstan wurden schon mehr als 400 Personen zeitweise festgenommen, weil sie gewisse Inhalte in sozialen Netzwerken gepostet oder geteilt hatten, insbesondere bei Facebook, VKontakte und Odnoklassniki. Sie wurden wegen der Aufstachelung zu nationalem, rassistischem, religiösem und sozialem Hass sowie zu Klassenhass strafrechtlich verfolgt. Die vagen Formulierungen in den Gesetzen zur Extremismusbekämpfung erlauben es der kasachstanischen Regierung, die gesetzlichen Vorschriften je nach Einzelfall zu interpretieren, um aktive Oppositionelle zu bestrafen. Außerdem haben die Regierungen eine Atmosphäre der Angst geschaffen. Insbesondere die Sicherheitsdienste in Kasachstan und Kirgistan nutzen diese juristischen Fallstricke, um das Gewaltmonopol des Staats zu stärken und 'potentieller Schläferzellen des IS bzw. deren Unterstützer zu verfolgen.

Im Prinzip haben es die zentralasiatischen Regierungen trotz aller Anstrengungen nicht geschafft, Terroristen an der Nutzung des Internets zu hindern. Außerdem hat ihr Kampf gegen den Extremismus zu Einschränkungen und Verletzungen von bürgerlichen Freiheitsrechten geführt.

\section{Bildung als wenig genutzte Möglichkeit}

Das dritte Instrument zur Abwehr der Propagierung von Extremismus im Internet ist Bildung. Traditionell hat zivilgesellschaftliches Engagement in Zentralasien andere swichtigere oder $`$ bedeutsamere Pflichten (z. B. im sozialen Bereich), als der Ausbreitung gewalttätigen Extremismus und Terrorismus durch die Stärkung der Resilienz lokaler Gemeinschaften entgegen zu wirken. Internationale Geldgeber besetzen diese Nische oft und unterstützen nichtstaatliche Akteure darin, lokale Projekte zur `Bekämpfung des gewaltbereiten Extremismus/
Terrorismus` (BGET) anzustoßen. Weder zivilgesellschaftliche Einrichtungen noch Think Tanks können sich ohne ausländische oder staatliche finanziellen Hilfen mit dem Thema des gewaltbereiten Extremismus und Terrorismus beschäftigen. Daher werden im Ergebnis meist projektgebundene Aktivitäten mit schnellen Ergebnissen statt langfristiger Strategien umgesetzt.

Hedajah, ein weltweites Zentrum zur Extremismusabwehr mit Hauptsitz in Saudi-Arabien, erprobt zum Beispiel zusammen mit der NGO Search for Common Ground (USA) ein Pilotprojekt für ein kulturell-literarisches BGET-Curriculum in Kirgistan, das das Bewusstsein für "gemeinsame BGET-Ansätze stärken und Regierungsbeamte und Vertreter der Zivilgesellschaft dazu befähigen soll, gewaltbereiten Extremismus zu verhindern bzw. ihm etwas entgegenzusetzen«. In Kasachstan lässt das UN-Büro für den Kampf gegen Drogen und Verbrechen (UNODC) zurzeit eine Reihe von Trainingseinheiten zum Thema 'Kampf gegen den Terrorismus ins Russische übersetzen, die im Rahmen der UNInitiative Education for Justice (E4J) entwickelt wurden. Sie sollen kasachstanischen Dozenten, die Kurse zum Thema Terrorismusbekämpfung an Universitäten und anderen wissenschaftlichen Einrichtungen anbieten, mit wichtigem, nützlichem Material versorgen.

Weder die aufklärenden noch die juristischen Instrumente befassen sich in ausreichendem Maße mit der Internetpräsenz des Extremismus in Zentralasien. Grundsätzlich herrscht Einigkeit darüber, dass Aufklärungskampagnen durchgeführt werden sollten, um weitere Radikalisierungen zu verhindern und den gewaltbereiten Extremismus und Terrorismus in der Region zu bekämpfen. Es gibt auch einige Internetseiten, die zu diesem Zweck geschaffen wurden. Zu diesen Plattformen gehört die neue Website www.capve. org, die im Mai 2018 auf Russisch und Englisch startete und von der Abteilung für Terrorismusprävention der UNODC und ihrem Regionalbüro in Zentralasien unterstützt wird. Diese Website wendet sich an Politiker und andere Experten, »die im Kampf gegen gewaltbereiten Extremismus aktiv sind " und soll wichtige Informationen, Forschungsergebnisse und andere Materialien zum Thema bereitstellen. Zurzeit kann man dort Dokumente der Vereinten Nationen, regionale Forschungsprojekte und -ergebnisse, Anleitungen zur Extremismusbekämpfung, nationale Programme, Gesetzesvorlagen u. a. finden. Die Website enthält zwar wichtige Informationen zum Thema, ist aber noch nicht besonders nutzerfreundlich. Sie führt zwar die weltweit besten Ansätze und Richtlinien zur Extremismusbekämpfung auf, stellt aber keinen Bezug zur Region Zentralasien her.

Während des österreichischen OSZE-Vorsitzes 2017 bot eine Social Media-Kampagne der OSZE mit dem 
Hashtag \#UnitedCVE jungen Menschen aus Zentralasien eine Plattform, um Ideen zu sammeln und ihre Erfahrungen und Wahrnehmungen von gewaltbereitem Extremismus, Radikalisierung und Terrorismus auszutauschen, das bisher Erreichte zu bewerten und Empfehlungen für politische Entscheidungsträger der OSZE in Wien auszusprechen. Dies war der erste Versuch, das Thema Extremismusbekämpfung mit Hilfe eines Hashtags anzugehen und Gegenerzählungen im Internet zu entwerfen und zu verbreiten. Von den angesprochenen Jugendlichen wurde diese Kampagne sehr gut aufgenommen.

\section{Fazit}

Abschließend muss betont werden, dass Medien eine zentrale Rolle bei einer effektiven Strategie zur Bekämpfung des Extremismus spielen müssen. Es ist bekannt, dass die Rahmenbedingungen für Medien in Zentralasien nicht sehr gut sind und nur wenige von ihnen unabhängig sind. Nur in Kirgistan ist die Medienlandschaft etwas vielfältiger. Die kasachstanische Regierung investiert beispielsweise regelmäßig Milliarden Tenge, um in den Massenmedien ihre Politik zu propagieren und kontrolliert auf diesem Wege alle Medien im Lande. Man sollte also annehmen, dass es ein Leichtes wäre, administrative wie finanzielle Ressourcen für den Aufbau einer Kommunikation über Terrorismusbekämpfung, die Verhinderung von Rekrutierungen über das Internet und für die Schaffung von Gegennarrativen aufzubringen. Kasachstan wie auch die anderen zentralasia- tischen Staaten sollten viel größere Anstrengungen in diesem Bereich unternehmen. Soziale Medien können für die Überwachung von Terrorismuspropaganda und bei der Stärkung der digitalen Resilienz viel wirksamer sein als die bloße Zensur des Internets.

Doch leider fehlt es den Regierungen der zentralasiatischen Staaten an Ressourcen, Vertrauen und, wichtiger noch, an Kreativität bei der Bekämpfung des Extremismus im Internet. Einerseits nehmen sie soziale Medien weiterhin als Bedrohung für die Stabilität ihrer Länder und für den Status quo wahr. Andererseits ist ihre einzige Methode, um das Problem des Extremismus im Internet anzugehen, immer noch die Blockierung und das Filtern bestimmter Plattformen und Websites. Auch weil die Regierungen der Öffentlichkeit das Thema nicht gut vermitteln, ist es sehr schwierig geworden, wirksame Gegendarstellungen und Narrative zu entwickeln, die im Internet verbreitet werden und gewalttätigem Extremismus vorbeugen können. Die zentralasiatischen Staaten ziehen es immer noch vor, sich altmodischer, nicht-internetbasierter Methoden zur Prävention und Bekämpfung von im Internet propagiertem gewaltbereitem Extremismus zu bedienen, statt mit Hilfe des Internets Antworten auf die neuen Herausforderungen zu kreieren. Um die Ergebnisse zu bewerten und die Chancen zu verstehen, die die Bekämpfung des gewaltbereiten Extremismus mit Hilfe von internetbasierten Methoden bietet, braucht es Zeit.

Aus dem Englischen von Brigitte Heuer

\section{Über die Autorin:}

Anna Gussarova ist Direktorin des Zentralasiatischen Instituts für Strategische Studien, Almaty, und Präsidentin der Vereinigung Kasachstanischer Alumni des Zentrums für Sicherheitsstudien des George C. Marshall-Zentrums. Zuvor war sie fünf Jahre lang Forschungsassistentin am Kasachstanischen Institut für Strategische Studien (KISI) unter dem Präsidenten der Republik Kasachstan. Ihre Forschungen beschäftigen sich mit transnationalen Sicherheitsfragen, Cybersicherheit, gewalttätigem Extremismus, Terrorismus sowie Extremismusbekämpfung und De-Radikalisierungsfragen.

\section{Lesetipps:}

- Kazakhstan to Spend \$840M on Countering Religious Extremism, Eurasianet, 6.3.2018, = <https://eurasianet. org/kazakhstan-to-spend-840m-on-countering-religious-extremism>

- Online Extremism in Central Asia: Repression is not Effective, But What's the Alternative?, SecDev.Foundation, 28.2.2017, = <https://www.secdev-foundation.org/online-extremism-central-asia-repression-not-effective-whatsalternative/>

- Noah Tucker, Public and State Responses to ISIS Messaging: Kazakhstan, CERIA Brief, No. 13, February 2016, $=<$ https://www.capve.org/components/com_jshopping/files/demo_products/CERIA_Brief_13_February_2016. $\mathrm{pdf}>$ 


\section{Zahl der Google-Suchanfragen zum Islamischen Staat in Zentralasien}

Grafik 1: Google-Suchanfragen zum IS in Kasachstan (im Zeitraum 11/2013-11/2018)

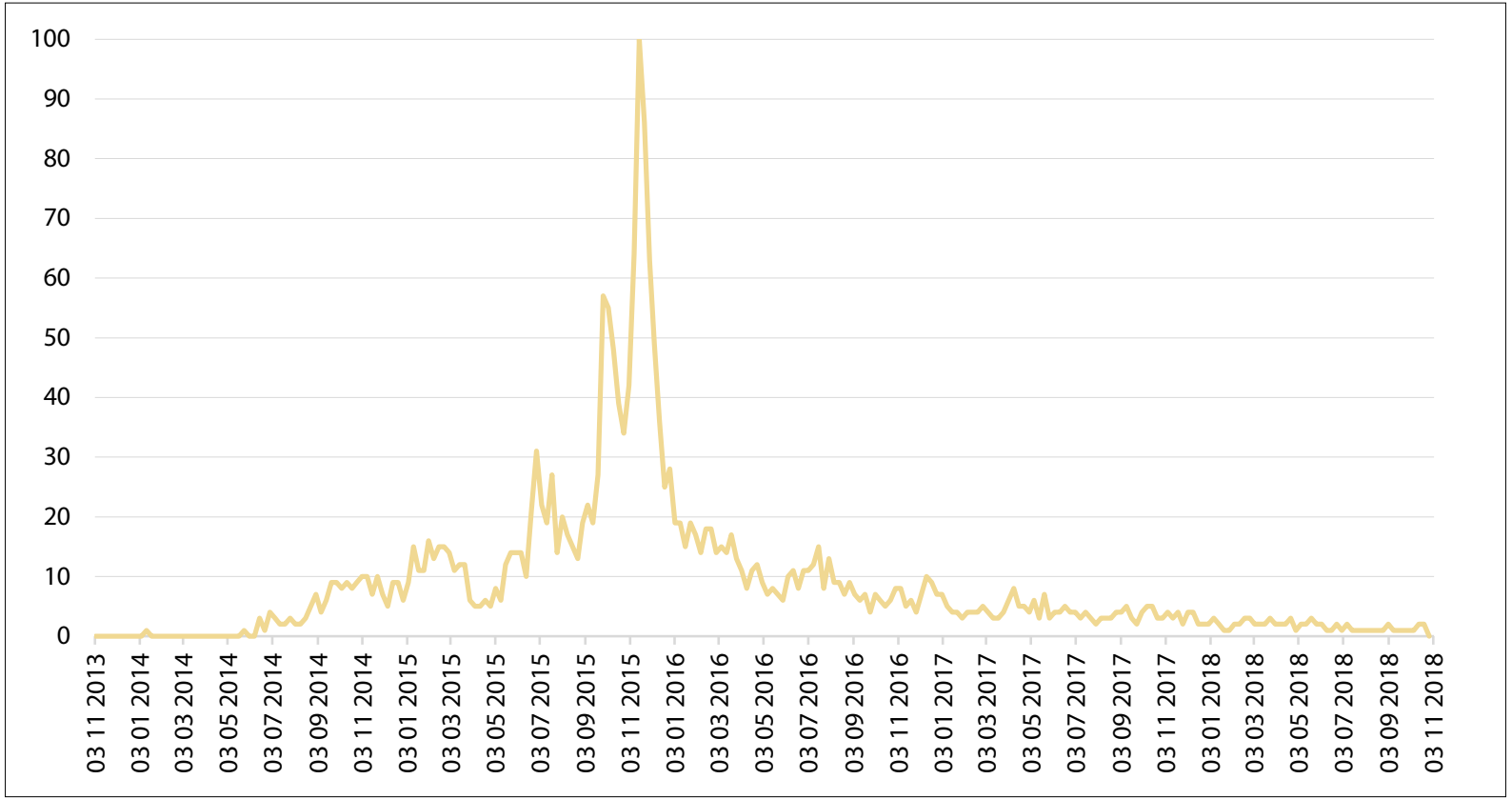

Quelle: Google Trends Analyse: Aufrufe für IS, Kasachstan <https://trends.google.com/trends/explore?date=today\%205-y\&geo=KZ\&q= \%D0\%B8\%D0\%B3\%D0\%B8\%D0\%BB>

Grafik 2: Google-Suchanfragen zum IS in Kirgistan (im Zeitraum 11/2013-11/2018)

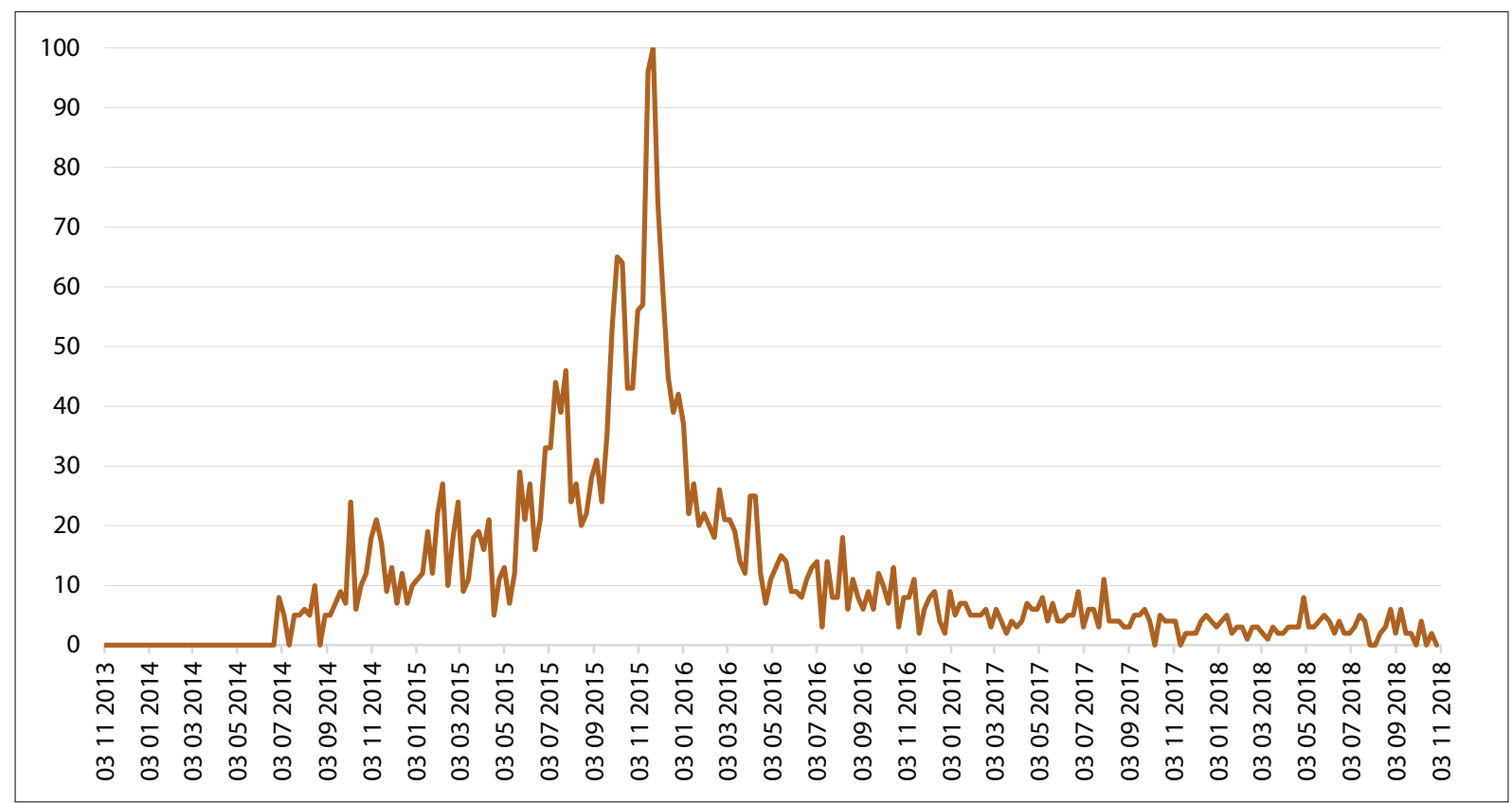

Quelle: Google Trends Analyse: Aufrufe für IS, Kirgistan <https://trends.google.com/trends/explore?date=today\%205-y\&geo=KG\&q= $\% \mathrm{D} 0 \% \mathrm{~B} 8 \% \mathrm{D} 0 \% \mathrm{~B} 3 \% \mathrm{D} 0 \% \mathrm{~B} 8 \% \mathrm{D} 0 \% \mathrm{BB}>$ 


\section{Grafik 3: Google-Suchanfragen zum IS in Tadschikistan (im Zeitraum 11/2013-11/2018)}

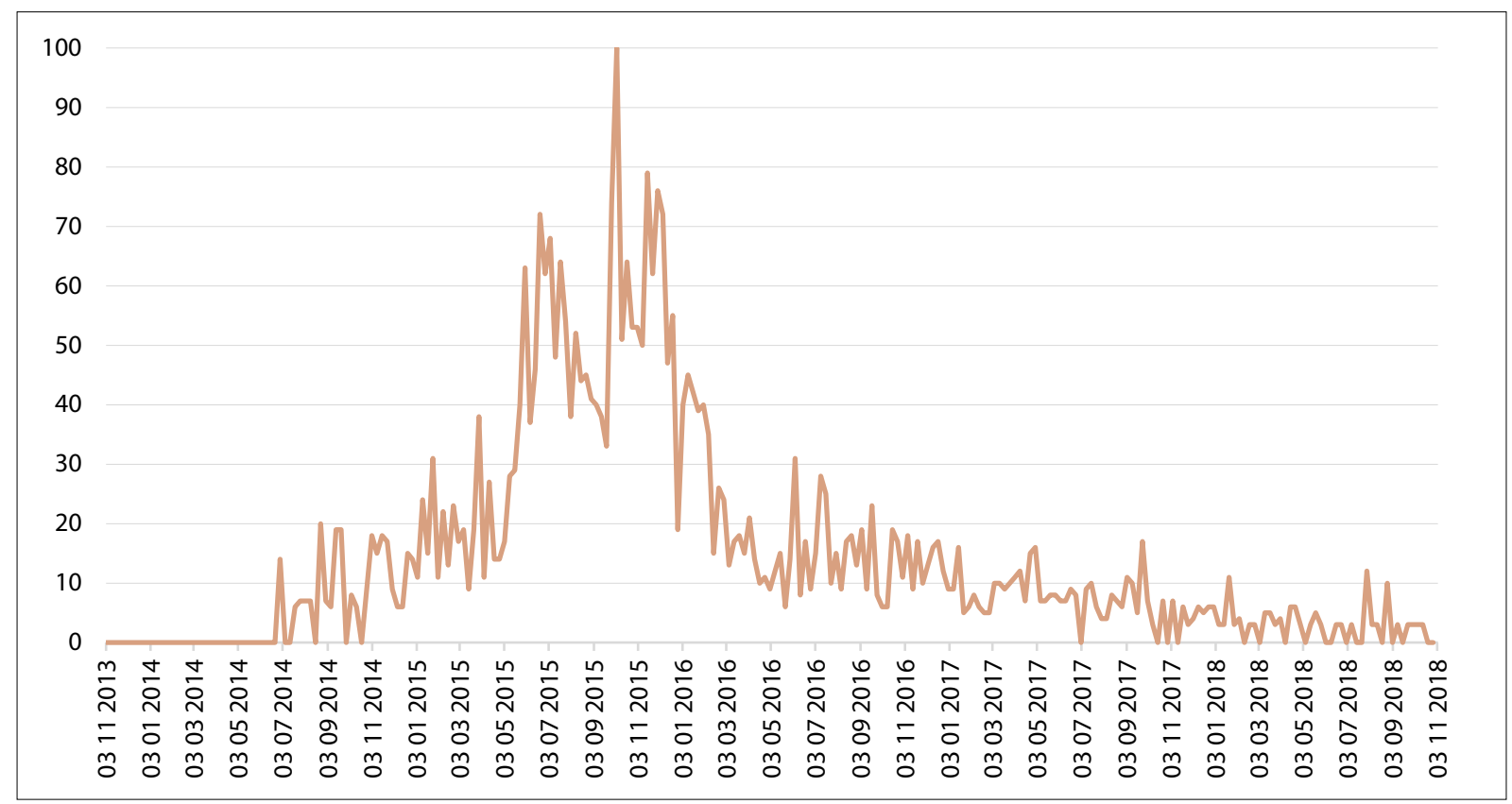

Quelle: Google Trends Analyse: Aufrufe für IS, Tadschikistan <https://trends.google.com/trends/explore?date=today\%205-y\&geo=TJ\&q= \%D0\%B8\%D0\%B3\%D0\%B8\%D0\%BB>

\section{Grafik 4: Google-Suchanfragen zum IS in Turkmenistan (im Zeitraum 11/2013-11/2018)}

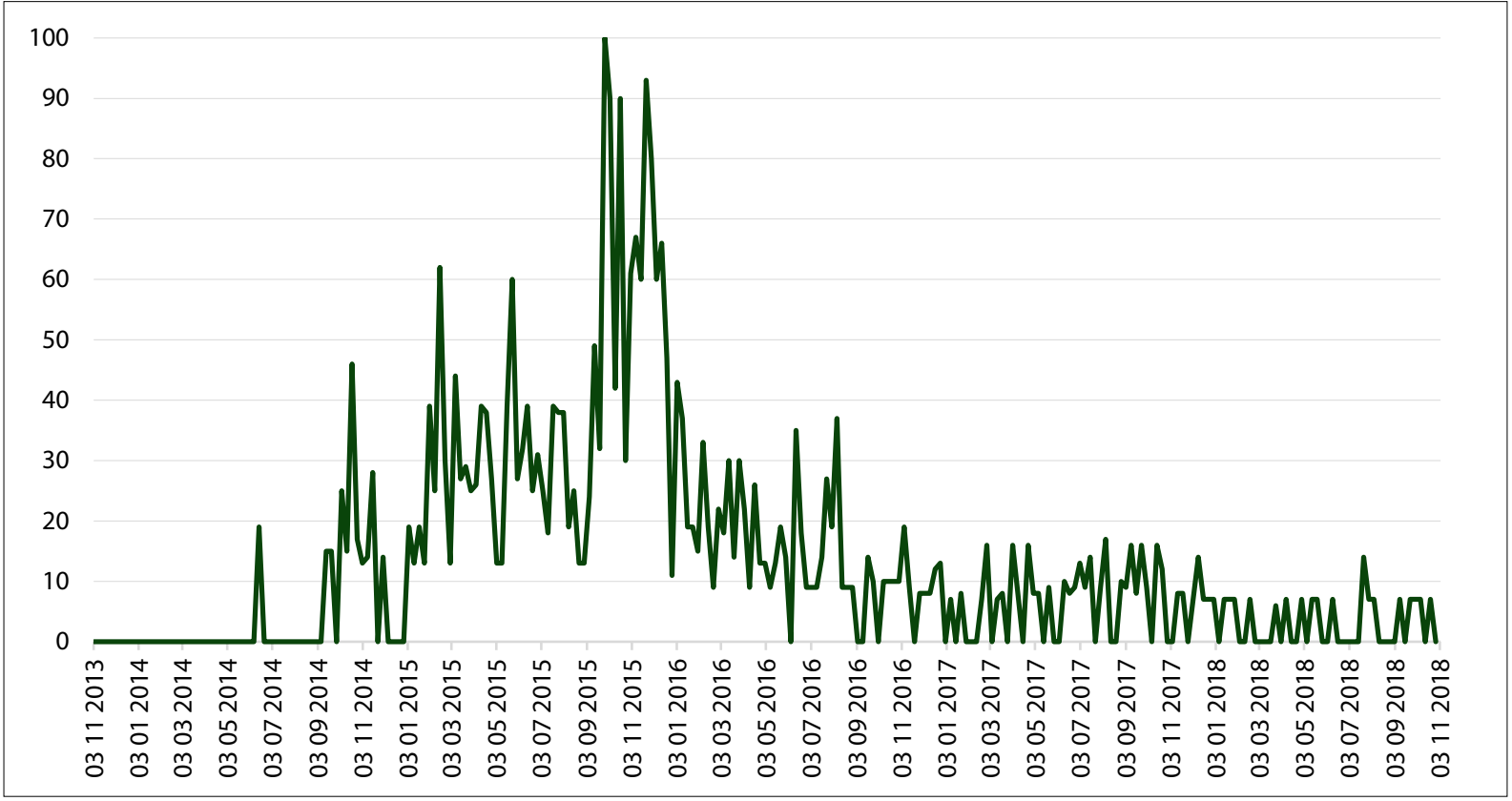

Quelle: Google Trends Analyse: Aufrufe für IS, Turkmenistan <https://trends.google.com/trends/explore?date=today\%205-y\&geo=TM\&q= $\% \mathrm{D} 0 \% \mathrm{~B} 8 \% \mathrm{D} 0 \% \mathrm{~B} 3 \% \mathrm{D} 0 \% \mathrm{~B} 8 \% \mathrm{D} 0 \% \mathrm{BB}>$ 
Grafik 5: Google-Suchanfragen zum IS in Usbekistan (im Zeitraum 11/2013-11/2018)

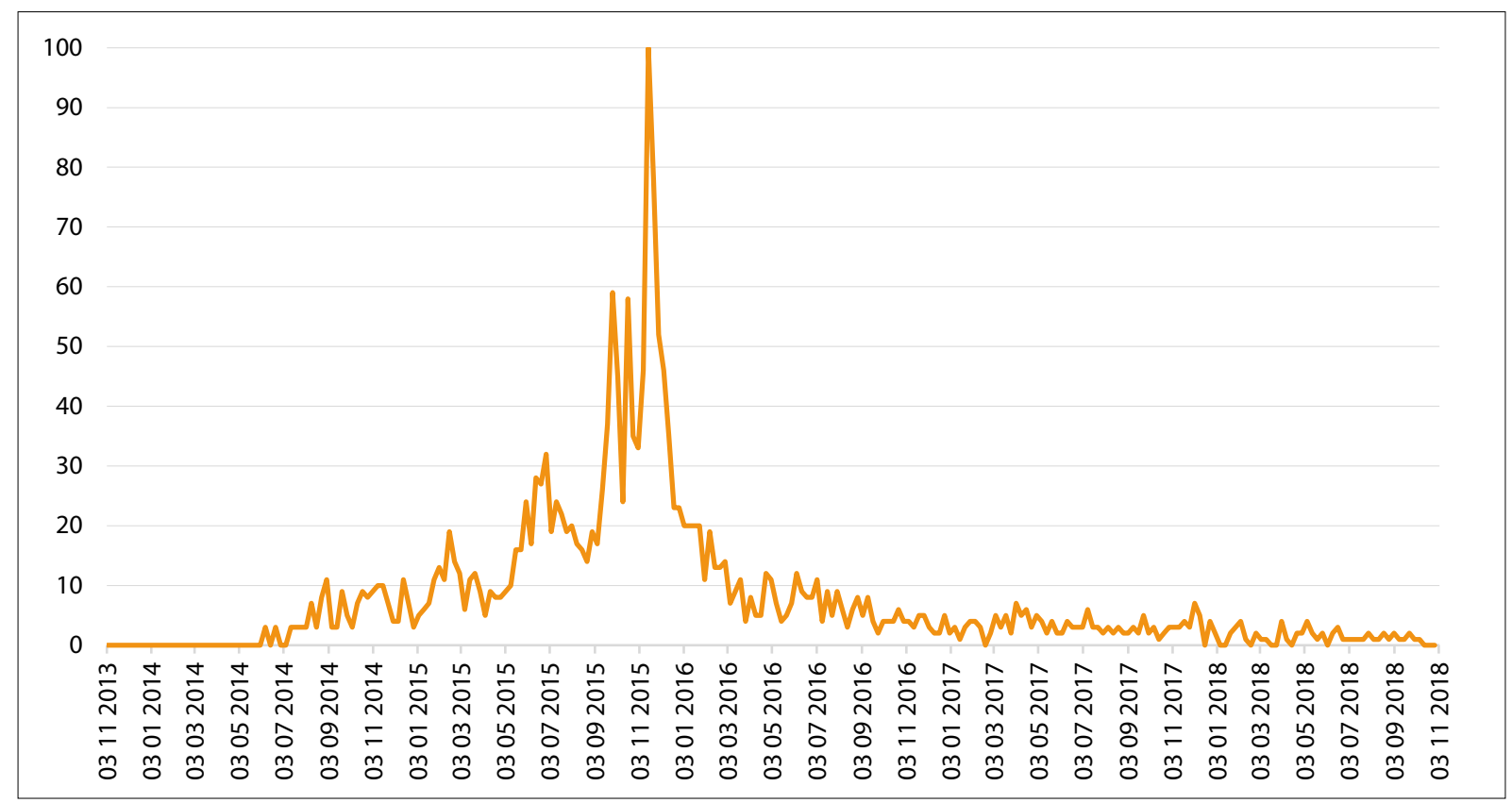

Quelle: Google Trends Analyse: Aufrufe für IS, Usbekistan <https://trends.google.com/trends/explore?date=today\%205-y\&geo=UZ\&q= \%D0\%B8\%D0\%B3\%D0\%B8\%D0\%BB>

\section{Meinungsumfragen in Kasachstan und Kirgistan zur Bekämpfung von Extremismus und Terrorismus}

Grafik 1: Wird der Kampf gegen den Terrorismus in Ihrem Land ihrer Meinung nach aktiv geführt? Meinungsumfrage in Kasachstan

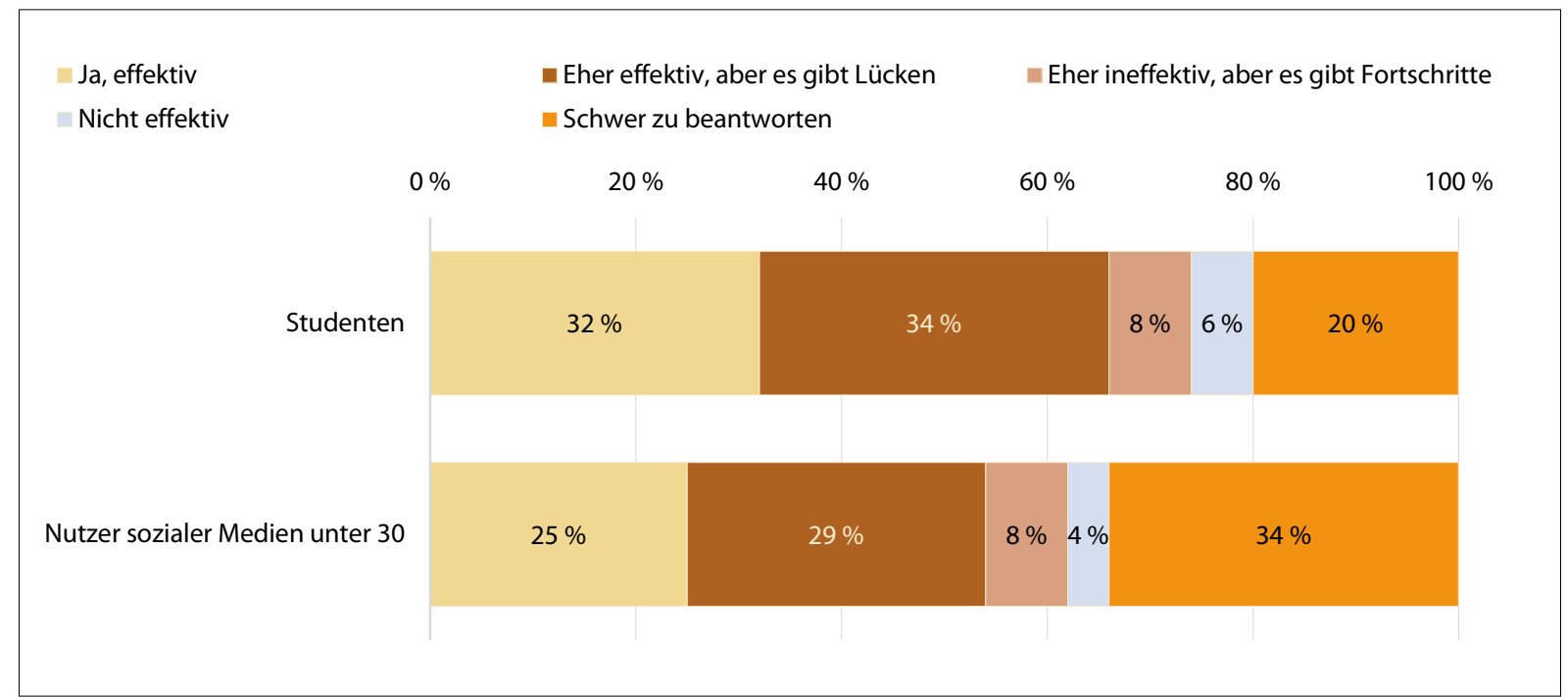

Die Umfrage erfolgte im Zeitraum von September 2015 bis Januar 2016 in Kasachstan. Insgesamt wurden 600 Respondenten befragt (davon 300 Studenten und 300 Nutzer sozialer Medien zwischen 18 und 30 Jahren, die einer Erwerbstätigkeit nachgehen).

Quelle: Institut für strategische Studien unter dem Präsidenten der Republik Kasachstan: Terrorismus und religiöser Extremismus in Zentralasien: Probleme der Wahrnehmung, Case Kasachstan und Kirgisistan, Reschetnjak A. (2016) <http://kisi.kz/uploads/33/files/6Rkxytkj.pdf> 
Grafik 2: Welche Maßnahmen müsste der Staat Ihrer Meinung nach gegen Terrorismus und religiösen Extremismus ergreifen? Meinungsumfrage in Kasachstan

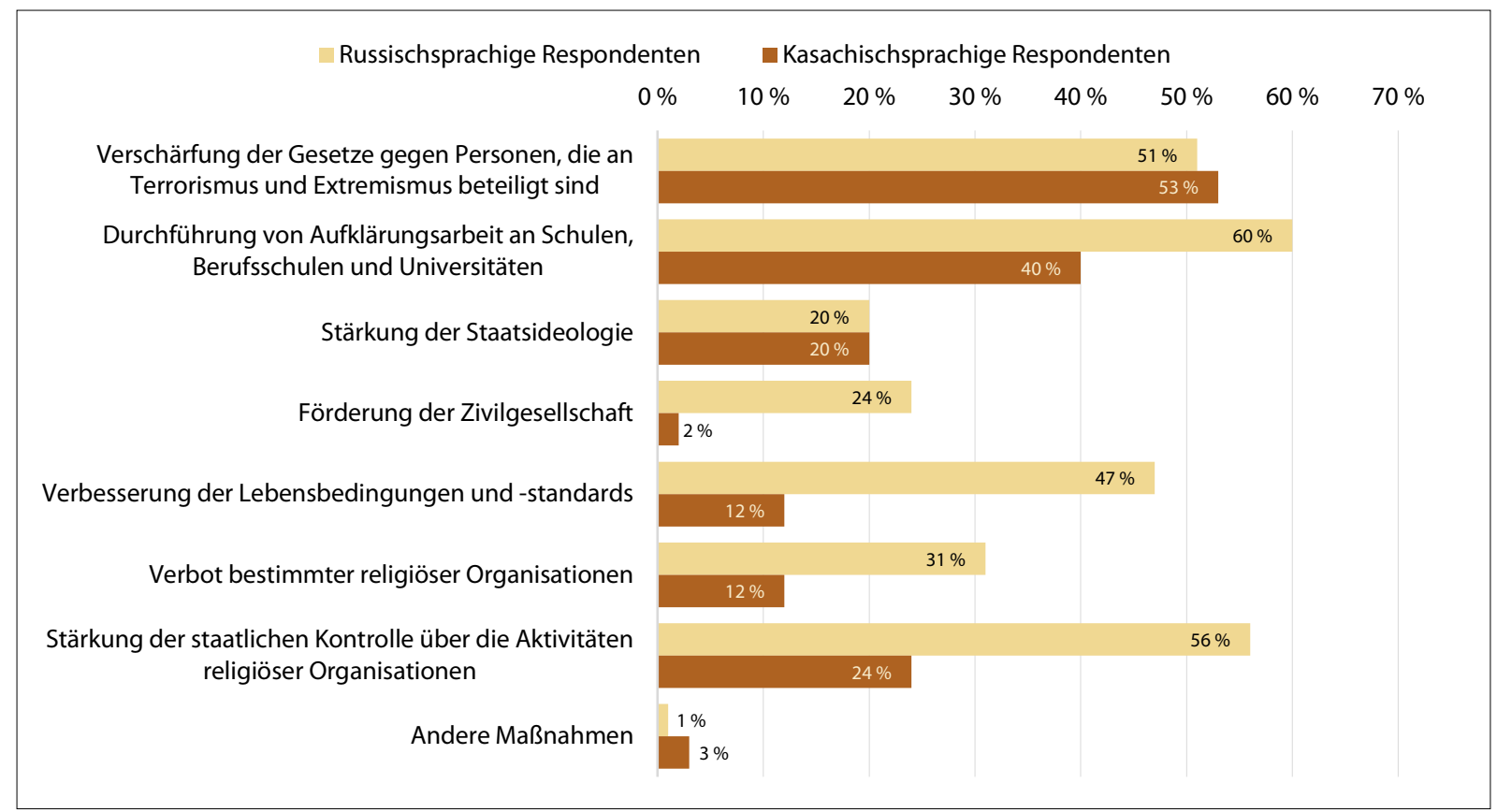

Die Umfrage erfolgte im Zeitraum von September 2015 bis Januar 2016 in Kasachstan. Insgesamt wurden 300 Respondenten zwischen 18 und 30 Jahren befragt. Quelle: Institut für strategische Studien unter dem Präsidenten der Republik Kasachstan: Terrorismus und religiöser Extremismus in Zentralasien: Probleme der Wahrnehmung, Case Kasachstan und Kirgisistan, Reschetnjak A. (2016) <http://kisi.kz/uploads/33/files/6Rkxytkj.pdf>

Grafik 3: Wird der Kampf gegen den Terrorismus in Ihrem Land ihrer Meinung nach aktiv geführt? Meinungsumfrage in Kirgistan

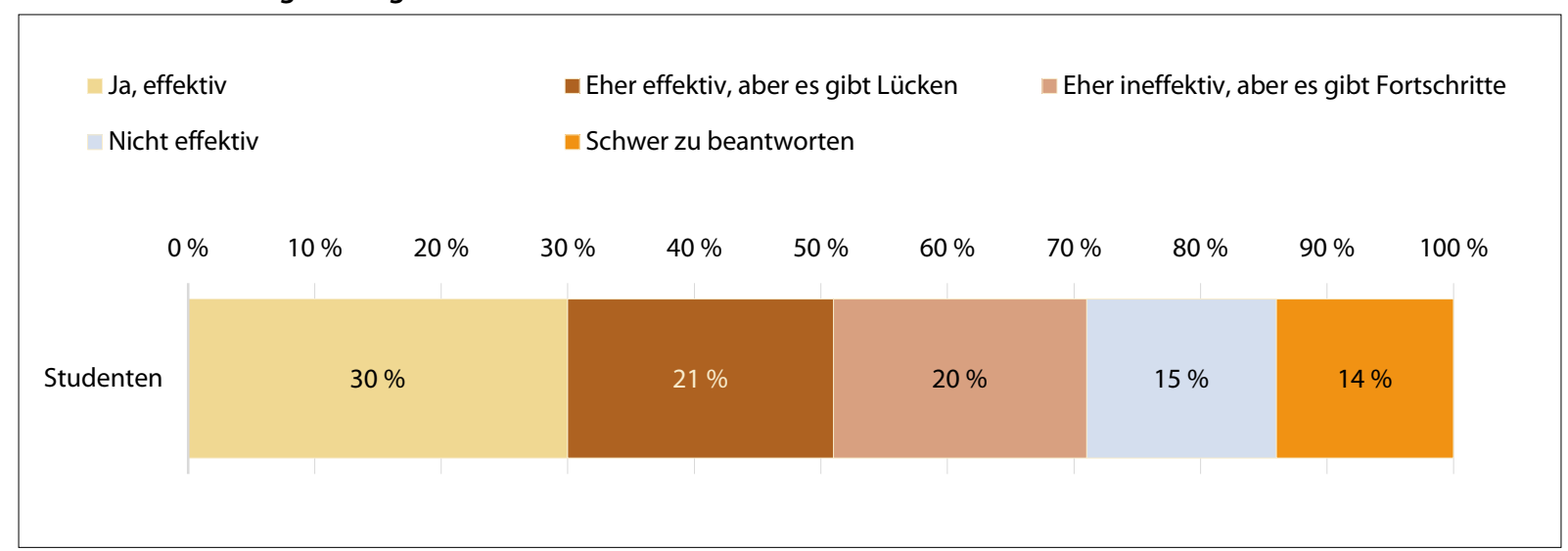

Die Umfrage erfolgte im Zeitraum von September 2015 bis Januar 2016 in Kirgistan. Insgesamt wurden 300 Studenten zwischen 18 und 30 Jahren befragt.

Quelle: Institut für strategische Studien unter dem Präsidenten der Republik Kasachstan: Terrorismus und religiöser Extremismus in Zentralasien: Probleme der Wahrnehmung, Case Kasachstan und Kirgisistan, Reschetnjak A. (2016) <http://kisi.kz/uploads/33/files/6Rkxytkj.pdf > 
Grafik 4: Welche Maßnahmen müsste der Staat Ihrer Meinung nach gegen Terrorismus und religiösen Extremismus ergreifen? Meinungsumfrage in Kirgistan

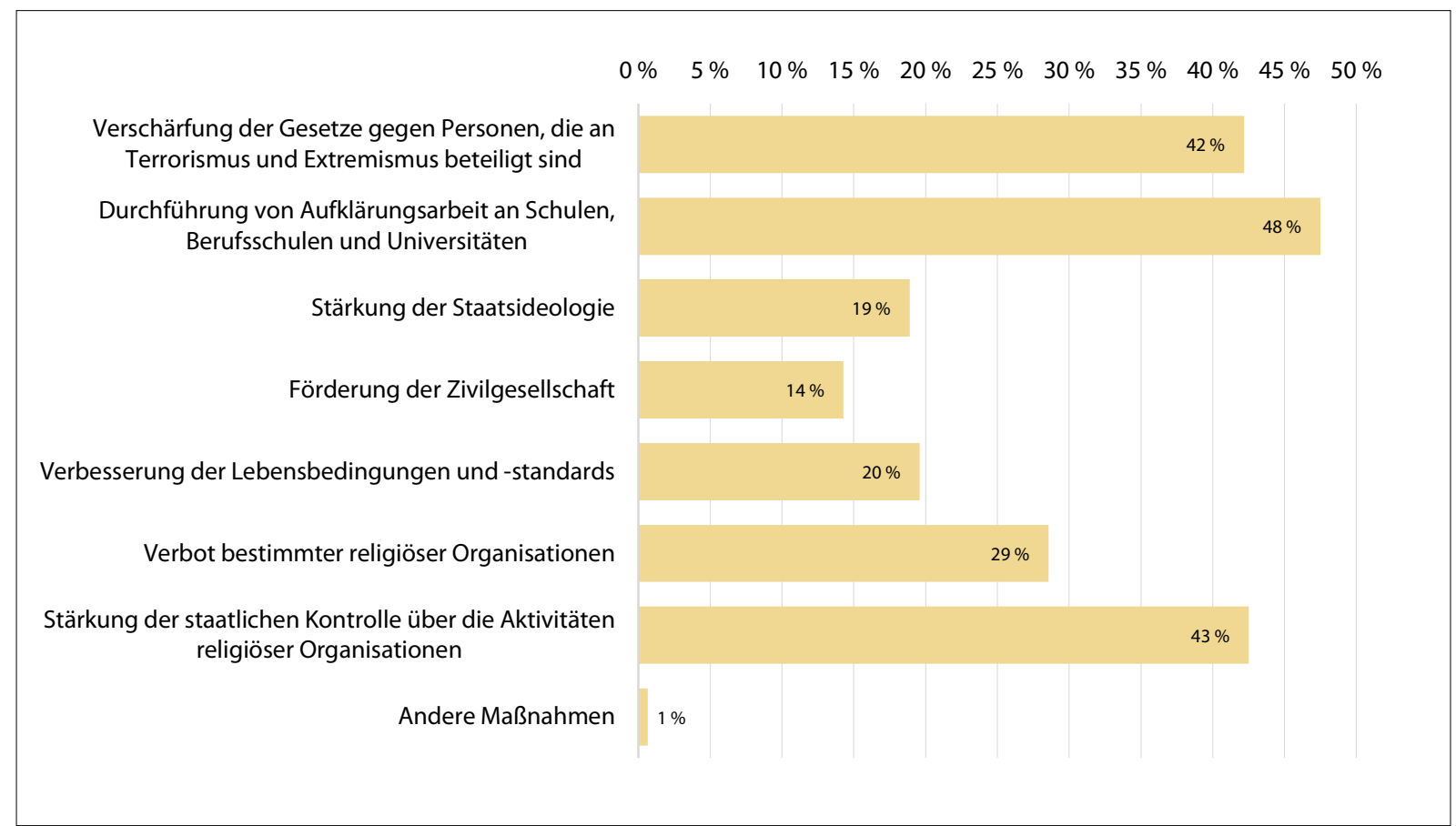

Die Umfrage erfolgte im Zeitraum von September 2015 bis Januar 2016 in Kirgistan. Insgesamt wurden 300 Respondenten zwischen 18 und 30 Jahren befragt. Quelle: Institut für strategische Studien unter dem Präsidenten der Republik Kasachstan: Terrorismus und religiöser Extremismus in Zentralasien:Probleme der Wahrnehmung, Case Kasachstan und Kirgisistan, Reschetnjak A. (2016) <http://kisi.kz/uploads/33/files/6Rkxytkj.pdf> 


\section{Oktober - 23. November 2018}

\section{Kasachstan}

\begin{tabular}{|c|c|}
\hline 20.10 .2018 & $\begin{array}{l}\text { Präsident Nursultan Nasarbajew trifft seine russischen und usbekischen Amtskollegen, Wladimir Putin und } \\
\text { Schawkat Mirsijojew, in Saryagasch (Gebiet Turkistan) zu informellen Beratungen über aktuelle Fragen der } \\
\text { Zusammenarbeit. }\end{array}$ \\
\hline 22.10 .2018 & $\begin{array}{l}\text { Der Geschäftsmann Iskander Jerimbetow, der im November } 2017 \text { verhaftet worden war, wird von einem Gericht } \\
\text { in Almaty wegen Korruption zu sieben Jahren Gefängnis verurteilt. Zwei seiner Mitarbeiter müssen für vier } \\
\text { Jahre und zwei Monate hinter Gitter. Angehörige beurteilen das Urteil als politisch motiviert. }\end{array}$ \\
\hline 24.10 .2018 & $\begin{array}{l}\text { Präsident Nasarbajew empfängt US-Handelsminister Wilbur Ross in Astana zu Gesprächen über den aktuel- } \\
\text { len Stand und die Perspektiven für die Entwicklung der Wirtschaftsbeziehungen zwischen beiden Ländern. }\end{array}$ \\
\hline 24.10 .2018 & $\begin{array}{l}\text { Dias Kadyrbajew, ein kasachischer Bekannter des Boston-Marathon-Bombers Dschochar Zarnajew, wird aus } \\
\text { einem US-amerikanischen Gefängnis entlassen und kehrt nach Kasachstan zurück. Kadyrbajew und zwei wei- } \\
\text { tere Freunde waren } 2015 \text { wegen Behinderung der Justiz zu mehreren Jahren Haft verurteilt worden, weil sie } \\
\text { Beweismaterial aus dem Zimmer des Attentäters entfernt hatten. }\end{array}$ \\
\hline 27.10 .2018 & $\begin{array}{l}\text { Außenminister Kairat Abdrachmanow empfängt den neuen kirgisischen Außenminister Tschingis Aidarbe- } \\
\text { kow in Astana zu Gesprächen über die bilateralen Beziehungen zwischen beiden Ländern. }\end{array}$ \\
\hline 28.10 .2018 & $\begin{array}{l}\text { Ein Regierungsbeschluss erlaubt es der Generalstaatsanwaltschaft, dem Staatlichen Komitee für nationale Sicher- } \\
\text { heit, dem Innenministerium und dem Verteidigungsministerium nun offiziell, Internetseiten in Notfallsitua- } \\
\text { tionen zu sperren, eine Praxis, die inoffiziell schon seit Jahren angewendet wird. }\end{array}$ \\
\hline 29.10 .2018 & $\begin{array}{l}\text { Tengrinews berichtet, dass das Staatliche Komitee für nationale Sicherheit am Wochenende im Rahmen einer } \\
\text { Spezialoperation im Gebiet Almaty sowie in drei weiteren Gebieten Kasachstans zehn Menschen festgenom- } \\
\text { men hat, denen terroristische Propaganda und Anstiftung zu religiösem Hass durch die Verbreitung von extre- } \\
\text { mistischen Inhalten in sozialen Netzwerken vorgeworfen wird. }\end{array}$ \\
\hline 30.10 .2018 & $\begin{array}{l}\text { Eine Parlamentsdelegation nimmt an der Plenarsitzung der Parlamentarischen Versammlung der CSTO in } \\
\text { Moskau teil. }\end{array}$ \\
\hline 30.10 .2018 & $\begin{array}{l}\text { Teile der Armee nehmen an militärischen Übungen der CSTO-Mitgliedstaaten unter dem Namen »Unzerstör- } \\
\text { bare Bruderschaft - } 2018 \text { « im russischen Jekaterinburg teil, an denen insgesamt } 1.300 \text { Soldaten beteiligt sind. }\end{array}$ \\
\hline 31.10 .2018 & $\begin{array}{l}120 \text { kasachstanische Soldaten, die sich an der UN Interim Force im Libanon (UNIFIL) als Teil des indischen } \\
\text { Bataillons Indbat beteiligen sollen, treffen in Beirut ein. Dies ist die erste kasachstanische Beteiligung an einer } \\
\text { Peacekeeping Mission der UN. }\end{array}$ \\
\hline 1.11 .2018 & $\begin{array}{l}\text { Präsident Nasarbajew empfängt den saudi-arabischen Innenminister Abdel Aziz bin Saud bin Naif in Astana } \\
\text { zu Gesprächen über die Ausweitung der Zusammenarbeit zwischen beiden Ländern. }\end{array}$ \\
\hline 2.11 .2018 & $\begin{array}{l}\text { In Astana findet ein Gipfeltreffen der Regierungschefs der GUS-Mitgliedsstaaten statt, an dem nicht nur Pre- } \\
\text { mierminister Bakytdschan Sagyntajew, sondern auch Präsident Nasarbajew teilnimmt. Am Rande des Gipfels } \\
\text { führt Nasarbajew bilaterale Gespräche mit dem russischen Premierminister Dmitrij Medwedjew. }\end{array}$ \\
\hline 2.11.2018 & $\begin{array}{l}\text { Rund } 50 \text { Kinder aus China stammender ethnischer Kasachen bitten bei einem Treffen im Kasachstanischen } \\
\text { Büro für Menschenrechte die Behörden um Unterstützung bei der Befreiung ihrer Eltern aus den sogenannten } \\
\text { Umerziehungslagern im Autonomen Gebiet Xinjiang der VR China. Nach ihren Angaben wurden ihre Eltern } \\
\text { bei regelmäßigen Reisen zwischen Kasachstan und China festgenommen. }\end{array}$ \\
\hline 5.11 .2018 & $\begin{array}{l}\text { Ein Gericht in Aktau (Gebiet Mangistau) stellt das Verfahren gegen den französischen Regisseur Vincent Prado } \\
\text { ein, der Ende September wegen des angeblichen Fehlens von Drehgenehmigungen für einen Film über die Pro- } \\
\text { teste von Schanaösen } 2011 \text { vorübergehend festgenommen und zu einer Geldstrafe verurteilt worden war. Prado } \\
\text { fordert eine finanzielle Entschädigung und eine offizielle Entschuldigung von den kasachstanischen Behörden. }\end{array}$ \\
\hline 6.11 .2018 & $\begin{array}{l}\text { Präsident Nasarbajew empfängt in Astana den estnischen Premierminister Jüri Ratas zu Gesprächen über eine } \\
\text { Intensivierung der Zusammenarbeit in den Bereichen Digitalisierung, Technologie und Innovationen. Am } \\
\text { Vortag hatte Ratas bereits Gespräche mit Premierminister Sagyntajew geführt und an der Eröffnungszeremo- } \\
\text { nie des neuen Technologieparks für IT-Start-ups »Astana Hub«teilgenommen. }\end{array}$ \\
\hline
\end{tabular}




\begin{tabular}{|c|c|}
\hline 8.11 .2018 & $\begin{array}{l}\text { In Astana findet ein Gipfeltreffen der CSTO-Mitgliedstaaten statt, bei dem sowohl die Staatsoberhäupter als } \\
\text { auch die Außenminister, Verteidigungsminister und die Vorsitzenden der nationalen Sicherheitsräte in verschie- } \\
\text { denen Formaten zusammenkommen, um Themen wie regionale Sicherheit und die Intensivierung der militäri- } \\
\text { schen Zusammenarbeit zu besprechen. Am Rande des Gipfels finden diverse bilaterale Treffen statt, u. a. zwi- } \\
\text { schen Verteidigungsminister Nurlan Jermekbajew und seinen Amtskollegen aus Armenien und Belarus, David } \\
\text { Tonojan und Andrej Rawkow. Die Staatsoberhäupter der sieben Mitgliedsstaaten unterzeichnen eine Reihe von } \\
\text { Dokumenten, u. a. zur Koordinierung der Maßnahmen im Kampf gegen den Terrorismus. }\end{array}$ \\
\hline 9.11 .2018 & $\begin{array}{l}\text { Ein Gericht in Aktobe verurteilt die drei Fahrer des Busses, der am 18.1.2018 auf der Fahrt von Schymkent in } \\
\text { das russischen Samara in Flammen aufgegangen war und } 54 \text { usbekische Gastarbeiter das Leben gekostet hatte, } \\
\text { zu fünf Jahren Haft. Zwei Mitarbeiter der Firma, der der Bus gehörte, werden wegen des Einsatzes eines tech- } \\
\text { nisch ungeeigneten Fahrzeugs zu jeweils dreieinhalb Jahren Arbeitskolonie verurteilt. }\end{array}$ \\
\hline 9.11 .2018 & $\begin{array}{l}\text { Präsident Nasarbajew empfängt seinen russischen Amtskollegen Putin im Rahmen des Interregionalen Koopera- } \\
\text { tionsforums Russland-Kasachstan in Petropawl (Gebiet Nordkasachstan), bei dem es u. a. um die Entwicklung } \\
\text { des Tourismus geht und ein gemeinsamer Aktionsplan für 2019-2021 sowie ein Plan für die interregionale und } \\
\text { grenzüberschreitende Zusammenarbeit zwischen Russland und Kasachstan 2018-2023 unterzeichnet werden. }\end{array}$ \\
\hline 12.11.2018 & $\begin{array}{l}\text { Dutzende Menschen gedenken auf einem Friedhof in Almaty des Oppositionspolitikers und Nasarbajew-Kri- } \\
\text { tikers Samanbek Nurkadilow, der vor } 13 \text { Jahren erschossen in seinem Haus aufgefunden wurde. Die Todes- } \\
\text { umstände sind bis heute umstritten, während offiziell von Selbstmord ausgegangen wird, nehmen Verwandte } \\
\text { und Oppositionspolitiker an, dass Nurkadilow ermordet wurde. }\end{array}$ \\
\hline 12.11 .2018 & $\begin{array}{l}\text { ußenminister Abdrachmanow und der chinesische Botschafter Zhang Xiao vereinbaren eine engere Zusam- } \\
\text { tenarbeit beim Schutz der ethnischen Kasachen im chinesischen Autonomen Gebiet Xinjiang. }\end{array}$ \\
\hline 13.11.2018 & $\begin{array}{l}\text { Am Rande der Konferenz »Astana Club« führt Präsident Nasarbajew Gespräche mit dem früheren afghani- } \\
\text { schen Präsidenten Hamid Karzai zu Fragen der regionalen Sicherheit um dem Konflikt in Afghanistan sowie } \\
\text { mit dem ehemaligen Präsidenten der Europäischen Kommission, Jose Manuel Barroso, über die Beziehungen } \\
\text { zwischen der EU und Kasachstan. }\end{array}$ \\
\hline 14.11 .2018 & $\begin{array}{l}\text { An allen Universitäten des Landes werden in einem 30-minütigen Test die Kenntnisse des lateinischen Alpha- } \\
\text { bets unter den Studenten überprüft. }\end{array}$ \\
\hline 14.11 .2018 & $\begin{array}{l}\text { Präsident Nasarbajew gibt den Start für den ersten Börsenhandel an der Börse des Astana International Finan- } \\
\text { cial Center (AIFC). }\end{array}$ \\
\hline 15.11 .2018 & $\begin{array}{l}\text { Präsident Nasarbajew und Premierminister Sagyntajew empfangen den slowakischen Premierminister Peter } \\
\text { Pellegrini in Astana zu Gesprächen über die Intensivierung der Handelsbeziehungen im Rahmen der chinesi- } \\
\text { schen Seidenstraßeninitiative. }\end{array}$ \\
\hline 15.11.2018 & $\begin{array}{l}\text { Die usbekische Oppositionsgruppe »Birdomlik Demokratische Volksbewegung» wird an der Durchführung } \\
\text { eines zweitägigen Kongresses in Schymkent gehindert, auf dem die Gründung einer Oppositionspartei geplant } \\
\text { war. Laut dem Führer der Bewegung, Bachodir Chorijew, wurde } 50 \text { Mitgliedern von den usbekischen Behör- } \\
\text { den die Ausreise verwehrt, während zehn weitere Mitglieder auf dem Weg von Almaty an der Weiterreise nach } \\
\text { Schymkent gehindert wurden. }\end{array}$ \\
\hline 16.11 .2018 & $\begin{array}{l}\text { asachstan stimmt als eines von } 26 \text { Ländern gegen einen Resolutionsentwurf des 3. Komitees der UN-Vollver- } \\
\text { mmlung, in dem Russland Menschenrechtsverletzungen auf der Krim vorgeworfen werden. }\end{array}$ \\
\hline 16.11 .2018 & $\begin{array}{l}\text { Der auf Betreiben Kasachstans international gesuchte ehemalige Bankier Dschormat Jertajew wird erneut in } \\
\text { Moskau verhaftet, nachdem ihm die russischen Behörden seinen Flüchtlingsstatus wieder entzogen hatten. }\end{array}$ \\
\hline 19.11.2018 & $\begin{array}{l}\text { Außenminister Abdrachmanow empfängt den Präsidenten der Parlamentarischen Versammlung der OSZE, } \\
\text { Giorgi Tsereteli, und den Sonderbeauftragten der OSZE für Zentralasien, Roman Haider, in Astana zu Gesprä- } \\
\text { chen über die Perspektiven der Kooperation und die Stärkung des interparlamentarischen Dialogs. }\end{array}$ \\
\hline 20.11 .2018 & $\begin{array}{l}\text { Außenminister Abdrachmanow führt in Astana Gespräche mit dem albanischen Minister für europäische und } \\
\text { auswärtige Angelegenheiten, Ditmir Buschati, über die Zusammenarbeit in der Bauindustrie, Energie und } \\
\text { Landwirtschaft sowie innerhalb der Organisation für Islamische Zusammenarbeit (OIC). }\end{array}$ \\
\hline 22.11 .2018 & $\begin{array}{l}\text { Am Rande des Kasachstan-Singapur Business Forums unterzeichnen der Minister für Investitionen und Ent- } \\
\text { wicklung, Dschenis Kassymbek, und der Staatsminister für Handel und Industrie von Singapur, Koh Poh } \\
\text { Koon, ein Investitionsabkommen. }\end{array}$ \\
\hline 22.11 .2018 & $\begin{array}{l}\text { Premierminister Sagnytajew wird bei einem offiziellen Staatsbesuch in China u. a. vom chinesischen Präsiden- } \\
\text { ten Xi Jinping empfangen. }\end{array}$ \\
\hline
\end{tabular}




\section{Kirgistan}

\begin{tabular}{|c|c|}
\hline 20.10 .2018 & $\begin{array}{l}\text { Der Berater und stellvertretende Leiter des Präsidentenbüros unter dem ehemaligen Präsidenten Almasbek } \\
\text { Atambajew, Ikramjan Ilmijanow, wird in Kasan (RF) festgenommen und umgehend dem kirgisischen Staatli- } \\
\text { chen Komitee für nationale Sicherheit (GKNB) übergeben, nachdem er am Vortag in Abwesenheit wegen Kor- } \\
\text { ruption angeklagt und zur Fahndung durch Interpol ausgeschrieben worden war. Ilmijanow hatte Kirgistan } \\
\text { im Juni } 2018 \text { mit unbekanntem Ziel verlassen. }\end{array}$ \\
\hline 22.10 .2018 & $\begin{array}{l}\text { Der GKNB verhaftet einen kirgisischen Staatsbürger aus Batken wegen der Verbreitung von Videoaufnahmen } \\
\text { und Nachrichten, die interethnische Konflikte schüren sollen, in sozialen Netzwerken. }\end{array}$ \\
\hline 25.10 .2018 & $\begin{array}{l}\text { Präsident Sooronbaj Dscheenbekow ernennt Ulukbek Kotschkorow zum neuen Minister für Arbeit und soziale } \\
\text { Entwicklung und Asamat Dschamankulow zum neuen Minister für Kultur, Information und Tourismus. }\end{array}$ \\
\hline 26.10 .2018 & $\begin{array}{l}\text { Bei einer Regierungssitzung fordert Präsident Dscheenbekow die Regierungsmitglieder auf, ihre oftmals »sinn- } \\
\text { losen« Dienstreisen ins Ausland massiv zu reduzieren. } 2018 \text { sollen Mitglieder der Regierung bereits mehr als } \\
400 \text { Dienstreisen unternommen haben. }\end{array}$ \\
\hline 27.10 .2018 & $\begin{array}{l}\text { Außenminister Tschingis Aidarbekow tauscht sich bei seinem ersten offiziellen Besuch in Astana mit seinem } \\
\text { kasachstanischen Amtskollegen Kairat Abdrachmanow über die bilateralen Beziehungen zwischen beiden Län- } \\
\text { dern aus. Am Tag zuvor hatte er bereits in Moskau mit dem russischen Außenminister Sergej Lawrow Fragen } \\
\text { der bilateralen Beziehungen und die Zusammenarbeit im Rahmen von EEU und CSTO erörtert. }\end{array}$ \\
\hline 29.10 .2018 & $\begin{array}{l}\text { In Fortsetzung seiner Antrittsbesuche in Partnerländern trifft Außenminister Aidarbekow in Tokio mit seinem } \\
\text { japanischen Amtskollegen Taro Kono zusammen. }\end{array}$ \\
\hline 29.10 .2018 & $\begin{array}{l}\text { Auf Einladung des türkischen Präsidenten Recep Tayyip Erdoğan nimmt Präsident Dscheenbekow an den Fei- } \\
\text { erlichkeiten zum 95. Jahrestag der Gründung der Republik Türkei in Istanbul teil. Am Rande der Feierlichkei- } \\
\text { ten führt Dscheenbekow bilaterale Gespräche mit seinem türkischen Amtskollegen sowie mit dem pakistani- } \\
\text { schen Präsidenten Arif Alvi. }\end{array}$ \\
\hline 30.10 .2018 & $\begin{array}{l}\text { Eine Parlamentsdelegation nimmt an der Plenarsitzung der Parlamentarischen Versammlung der CSTO in } \\
\text { Moskau teil. }\end{array}$ \\
\hline 30.10 .2018 & $\begin{array}{l}\text { Teile der Armee nehmen an militärischen Übungen der CSTO-Mitgliedsstaaten unter dem Namen »Unzer- } \\
\text { störbare Bruderschaft - } 2018 \text { « in Jekaterinburg (RF) teil, an dem insgesamt } 1.300 \text { Soldaten beteiligt sind. }\end{array}$ \\
\hline 31.10 .2018 & $\begin{array}{l}\text { Der GKNB gibt bekannt, dass ein kirgisischer Staatsbürger wegen der Überweisung von } 100.000 \text { US-Dollar } \\
\text { an terroristische Gruppen in Syrien verhaftet wurde. }\end{array}$ \\
\hline 2.11 .2018 & $\begin{array}{l}\text { Premierminister Muchammedkalyi Abylgasijew nimmt an einem Gipfeltreffen der Regierungschefs der GUS- } \\
\text { Mitgliedsstaaten in Astana teil. }\end{array}$ \\
\hline 2.11 .2018 & $\begin{array}{l}\text { Der GKNB gibt bekannt, dass im Gebiet Tschui fünf Ausländer und vier kirgisische Staatsbürger wegen des } \\
\text { Verdachts auf Mitgliedschaft in einer terroristischen Vereinigung festgenommen wurden. }\end{array}$ \\
\hline 7.11 .2018 & $\begin{array}{l}\text { Außenminister Aidarbekow führt im Vorfeld eines Treffens der CSTO-Mitgliedsstaaten in Astana Gespräche } \\
\text { mit seinem tadschikischen Amtskollegen Sirodschiddin Muchriddin (Aslow) über die Zusammenarbeit der } \\
\text { beiden Länder in Außenpolitik, Handel und Wirtschaft, Kultur und Grenzsicherung. }\end{array}$ \\
\hline 7.11 .2018 & $\begin{array}{l}\text { Der Bürgerbeauftragte Tokon Mamytow und die russische Beauftragte für Kinder, Anna Kusnezowa, unter- } \\
\text { zeichnen in Moskau ein MoU über die Zusammenarbeit im Bereich Schutz der Rechte und der Interessen von } \\
\text { Kindern. }\end{array}$ \\
\hline 8.11 .2018 & $\begin{array}{l}\text { Präsident Dscheenbekow, Außenminister Aidarbekow und Generalstabschef Rajimberdi Duischenbijew neh- } \\
\text { men am Gipfeltreffen der CSTO-Mitgliedstaaten in Astana teil, bei dem u. a. die Verbesserung der militäri- } \\
\text { schen Zusammenarbeit, die Aktivitäten des Krisenreaktionszentrums und Fragen der regionalen Sicherheit in } \\
\text { den unterschiedlichen Organen der Organisation besprochen werden. Am Rande des Gipfels finden diverse } \\
\text { bilaterale Treffen statt, u. a. zwischen Außenminister Aidarbekow und seinen Amtskollegen aus Tadschikistan } \\
\text { und Belarus, Muchriddin und Wladimir Makej. }\end{array}$ \\
\hline 9.11 .2018 & $\begin{array}{l}\text { In Dschalalabad wird die Journalistin Sanrabia Satybaldijewa vor ihrem Haus von unbekannten Tätern nie- } \\
\text { dergestochen und schwer verletzt. }\end{array}$ \\
\hline 10.11 .2018 & $\begin{array}{l}\text { Innenminister Kaschkar Dschunuschalijew wird in Moskau von seinem russischen Amtskollegen Wladimir } \\
\text { Kolokolzew zu Gesprächen über die Zusammenarbeit in der Kriminalitätsbekämpfung empfangen. }\end{array}$ \\
\hline 13.11.2018 & $\begin{array}{l}\text { Eine Delegation des Parlaments nimmt an den Feierlichkeiten zum 100. Jahrestag des Endes des Ersten Welt- } \\
\text { kriegs in Frankreich teil. Am Rande findet ein kurzes Treffen zwischen Parlamentssprecher Dastanbek Dschu- } \\
\text { mabekow und dem französischen Präsidenten Emmanuel Macron statt. }\end{array}$ \\
\hline
\end{tabular}




\begin{tabular}{|c|c|}
\hline 14.11 & $\begin{array}{l}\text { Nach Angaben des Statistikamts der RF sind in den ersten zehn Monaten } 2018 \text { nur } 247.600 \text { kirgisische Arbeits- } \\
\text { migranten nach Russland neu eingereist. Im Vorjahr waren es im gleichen Zeitraum mit } 472.000 \text { fast dop- } \\
\text { pelt so viele. }\end{array}$ \\
\hline 14.11 .2018 & $\begin{array}{l}\text { Eine militärische Delegation unter Leitung von Generalstabschefs Duischenbijew führt in Ankara Gespräche } \\
\text { über die militärische Zusammenarbeit mit dem Generalstabschef der türkischen Streitkräfte, Yasar Güler, und } \\
\text { dem türkischen Verteidigungsminister Hulusi Akar. }\end{array}$ \\
\hline 16.11.2018 & $\begin{array}{l}\text { In einem Interview mit der Nachrichtenagentur 24.kg beschuldigt Präsident Dscheenbekow seinen Vorgänger } \\
\text { Atambajew der Einflussnahme und der Ausübung von Druck auf seine Person und Politik. }\end{array}$ \\
\hline 16.11 .2018 & $\begin{array}{l}\text { irgistan stimmt als eines von } 26 \text { Ländern gegen einen Resolutionsentwurf des 3. Komitees der UN-Vollver- } \\
\text { mmlung, in dem Russland Menschenrechtsverletzungen auf der Krim vorgeworfen werden. }\end{array}$ \\
\hline 16.11 .2018 & $\begin{array}{l}\text { äsident Dscheenbekow empfängt den Sprecher des usbekischen Parlaments, Nuridschon Ismoilow, zu Gesprä- } \\
\text { en über das bilaterale Verhältnis. }\end{array}$ \\
\hline 18.11 & $\begin{array}{l}\text { Bei einer Sitzung des Ständigen Rats der CSTO wird der Vorsitz der Organisation von Kasachstan an Kirgistan } \\
\text { übergeben und Aman Mambetsejtow, Bevollmächtigter Kirgistans bei der CSTO, einstimmig als neuer Vor- } \\
\text { sitzender des Ständigen Rates bestätigt. }\end{array}$ \\
\hline 19.11 & $\begin{array}{l}\text { In einem Interview mit dem Fernsehkanal April wirft Ex-Präsident Atambajew seinem Nachfolger Dscheen- } \\
\text { bekow vor, dem Beispiel des } 2010 \text { entmachteten Präsidenten Kurmanbek Bakijew zu folgen, in dem er Klien- } \\
\text { telismus und Nepotismus fördere und unbegründete Festnahmen anordne. }\end{array}$ \\
\hline 21.11 & $\begin{array}{l}\text { Außenminister Aidarbekow führt in Vilnius Gespräche mit seinem litauischen Amtskollegen Linas Linkevičius } \\
\text { über die bilaterale Zusammenarbeit. }\end{array}$ \\
\hline 21.11 & $\begin{array}{l}\text { Präsident Dscheenbekow diskutiert auf einer Konferenz mit den bisherigen Parlamentssprechern und Premier- } \\
\text { ministern des Landes, außer denjenigen der Amtszeiten Bakijews und Atambajews, die politische Entwicklung } \\
\text { des Landes, Justiz- und Verfassungsreformen, Änderungen im Wahlsystem und wirtschaftliche Großprojekte. }\end{array}$ \\
\hline 22.11 & $\begin{array}{l}\text { ine Parlamentsdelegation unter Leitung von Parlamentssprecher Dschumabekow nimmt an der Plenarsit- } \\
\text { ung der der Parlamentarischen Versammlung der turksprachigen Länder (TurkPA) im türkischen Izmir teil. }\end{array}$ \\
\hline 22.11.2018 & $\begin{array}{l}\text { In Bischkek wird der Vorstandsvorsitzende des Verlags Wetschernyj Bischkek, Aleksandr Rjabuschkin, wegen } \\
\text { nicht näher definierter Gesetzesverletzungen verhaftet. }\end{array}$ \\
\hline 22.11.2018 & $\begin{array}{l}\text { Die Präsidialadministration teilt mit, dass ein Rat für die Beziehungen zu ausländischen Mitbürgern gegrün- } \\
\text { det wurde, um auf die wachsende Zahl der Kirgisen im Ausland zu reagieren und Investitionen zu fördern. }\end{array}$ \\
\hline 23.11.2018 & $\begin{array}{l}\text { Außenminister Aidarbekow trifft in Brüssel u. a. mit Vertretern des EU-Parlaments und mit der Hohen Ver- } \\
\text { treterin der EU für Außen- und Sicherheitspolitik, Federica Mogherini, zu Gesprächen über eine Vertiefung } \\
\text { der gegenseitigen Beziehungen zusammen. }\end{array}$ \\
\hline 23.11 .2018 & In der Stadt Osch nehmen Tausende an einer Demonstration gegen Extremismus und Terrorismus teil. \\
\hline
\end{tabular}

\section{Tadschikistan}

\begin{tabular}{|l|l|}
\hline 22.10 .2018 & $\begin{array}{l}\text { In der Nacht wird ein 23-jähriger tadschikischer Staatsbürger im russischen Sankt Petersburg von drei Unbe- } \\
\text { kannten durch Schläge und Schüsse so schwer verletzt, dass er operiert werden muss. }\end{array}$ \\
\hline 22.10.2018 & $\begin{array}{l}\text { Das Innenministerium garantiert heimkehrwilligen Bürgern, die in Syrien oder dem Irak auf Seiten terroris- } \\
\text { tischer und extremistischer Gruppen gekämpft haben, Rechtsbeistand. Betroffene können sich vorab telefo- } \\
\text { nisch oder über die sozialen Netzwerke an die Verwaltung für den Kampf gegen das organisierte Verbrechen } \\
\text { wenden und um Überprüfung ihres Falles bitten. }\end{array}$ \\
\hline 23.10 .2018 & $\begin{array}{l}\text { Im Untersuchungsgefängnis Nr. 1 von Duschanbe beginnt unter Ausschluss der Medien der Prozess gegen } 17 \\
\text { des Attentats auf ausländische Radfahrer in Dangara (Gebiet Chatlon) im Juli 2018 Verdächtige. Nur einer } \\
\text { von ihnen, Chusein Abdusamadow, war am Überfall selbst beteiligt, die anderen Angeklagten sind der Zuge- } \\
\text { hörigkeit zu einer terroristischen Vereinigung und des Verschweigens einer Straftat angeklagt. }\end{array}$ \\
\hline 23.10.2018 & $\begin{array}{l}\text { Tadschikische Medien berichten wiederholt, dass seit mehreren Wochen in manchen Bezirken des Landes der } \\
\text { Strom rationiert sei. Das staatliche Energieversorgungsunternehmen Barki totschik erklärt dies mit Reparatur- } \\
\text { maßnahmen, unabhängige Beobachter sehen eher einen Zusammenhang mit den hohen Stromexporten bzw. } \\
\text { der bevorstehenden Inbetriebnahme des Wasserkraftwerkes von Rogun. }\end{array}$ \\
\hline
\end{tabular}




\begin{tabular}{|c|c|}
\hline 23.10 .2018 & $\begin{array}{l}\text { Außenminister Sirodschiddin Muchriddin (Aslow) empfängt im Vorfeld der jährlichen bilateralen Konsulta- } \\
\text { tionen U.S. Deputy Assistant Secretary for Central Asia and Press and Public Diplomacy, Emilia Puma, zu } \\
\text { Gesprächen über aktuelle politische und ökonomische Fragen. Puma erklärt später vor Journalisten, die USA } \\
\text { seien besorgt über die Lage der Menschenrechte in Tadschikistan und hätten die Regierung des Landes auf- } \\
\text { gefordert, den Fall des aus politischen Gründen zu einer langjährigen Haftstrafe verurteilten Anwalts Busurg- } \\
\text { mechr Jorow zu überprüfen. }\end{array}$ \\
\hline 24.10 .2018 & $\begin{array}{l}\text { Der Sprecher der oberen Kammer des Parlamentes, Machmadsaid Ubajdullojew, wird in Moskau vom Spre- } \\
\text { cher der Staatsduma, Wjatscheslaw Wolodin, zu Gesprächen u. a. über Möglichkeiten der Verbesserung der } \\
\text { sozialen Situation der tadschikischen Arbeitsmigranten in Russland empfangen. }\end{array}$ \\
\hline 24.10 .2018 & $\begin{array}{l}\text { Ein Gericht in Duschanbe verurteilt den Leiter einer Abteilung der Verwaltung für den Kampf gegen illega- } \\
\text { len Drogenhandel, der im August } 2018 \mathrm{im} \text { Moment der Annahme von Bestechungsgeld in Höhe von } 20.000 \\
\text { US-Dollar festgenommen worden war, zu einer Geldstrafe von } 80.000 \text { Somoni (8.700 US-Dollar) und verfügt } \\
\text { seine Freilassung aus der Untersuchungshaft. }\end{array}$ \\
\hline 26.10 .2018 & $\begin{array}{l}\text { In Duschanbe findet der 10. Menschenrechtsdialog zwischen der EU und Tadschikistan statt. Die EU-Vertre- } \\
\text { ter begrüßen die Freilassung von Chajrullo Mirsaidow und Schuchrat Kudratow, rufen die tadschikische Füh- } \\
\text { rung aber u. a. zur verstärkten Untersuchung von Foltervorwürfen auf. }\end{array}$ \\
\hline 26.10 .2018 & $\begin{array}{l}\text { Bei einem Treffen mit Journalisten fordert Präsident Emomali Rachmon die Medienvertreter auf, weniger Lobes- } \\
\text { meldungen zu publizieren, da sie einen falschen Eindruck erwecken und Politiker zu Fehlern verleiten würden. }\end{array}$ \\
\hline 27.10 .2018 & $\begin{array}{l}\text { Rachmatullo Zoirow wird vom Parteitag der Sozialdemokraten (SDPT) zu ihrem Vorsitzenden wiedergewählt. } \\
\text { Die von Delegierten vorgeschlagenen Gegenkandidaten hatten ihre Kandidatur vor der Wahl zurückgezogen. }\end{array}$ \\
\hline 28.10 .2018 & $\begin{array}{l}\text { Der tadschikische Dienst von RFE/RL meldet unter Berufung auf eine Quelle beim Obersten Gericht, dass } \\
\text { der ehemalige Chef der Verwaltung für den Kampf gegen illegalen Drogenhandel beim Innenministerium, } \\
\text { Fajsullo Gadojew, bereits Mitte des Monats in absentia zu einer 28-jährigen Gefängnisstrafe verurteilt wurde. }\end{array}$ \\
\hline 29.10 .2018 & $\begin{array}{l}\text { Ein tadschikischer Staatsbürger startet auf Change.org eine Onlinepetition, in der gefordert wird, dass Staats- } \\
\text { diener und ihre Angehörigen keine Auslandskonten führen dürfen und ihre Urlaube im Land verbringen müs- } \\
\text { sen (siehe: <https://www.change.org/p/эмомали-рахмон-таджистан-чиновники-тоже-должны-придержив } \\
\text { аться-закона>). }\end{array}$ \\
\hline 29.10 .2018 & $\begin{array}{l}\text { Nach Angaben des Komitees zur Verteidigung politischer Gefangener in Tadschikistan wurden die beiden } \\
\text { Führungsmitglieder der Partei der Islamischen Wiedergeburt (PIWT) Muchammadali Fajsmuchammad und } \\
\text { Dschaloliddin Machmud nach durch Folter bedingter Verschlechterung ihres Gesundheitszustandes in ein } \\
\text { Krankenhaus überführt. }\end{array}$ \\
\hline 30.10 .2018 & $\begin{array}{l}\text { Die BBC berichtet unter Berufung auf eine offizielle iranische Website, dass das Wirtschaftsgericht Tadschikistans } \\
\text { auf Antrag des Staatlichen Komitees für Investitionen und die Verwaltung des Staatsbesitzes die Beendigung der } \\
\text { Tätigkeit von mehr als } 20 \text { Unternehmen iranischer Staatsbürger beschlossen hat. Gründe seien nicht bekannt. }\end{array}$ \\
\hline 31.10 .2018 & $\begin{array}{l}\text { Im neuesten Doing Business Index } 2019 \text { nimmt Tadschikistan Rang } 126 \text { von } 190 \text { Staaten ein und rutscht damit } \\
\text { gegenüber dem Vorjahr um drei Plätze ab. }\end{array}$ \\
\hline 2.11 .2018 & $\begin{array}{l}\text { Der Vorsitzende der Liberaldemokratischen Partei Russland, Wladimir Schirinowskij, wird von Präsident Rach- } \\
\text { mon zu vorher nicht geplanten Gesprächen über die Zusammenarbeit beider Staaten empfangen. Schirinow- } \\
\text { skij war am Vortag bereits mit Außenminister Muchriddin zusammengetroffen und hatte sich vor der Presse } \\
\text { sehr positiv über Präsident Rachmon geäußert. }\end{array}$ \\
\hline 2.11.2018 & $\begin{array}{l}\text { Präsident Rachmon empfängt den saudischen Innenminister, Prinz Abdel Asis bin Saud bin Najef, zu Gesprä- } \\
\text { chen über Stand und Perspektiven der Zusammenarbeit. }\end{array}$ \\
\hline 2.11 .2018 & $\begin{array}{l}\text { Premierminister Kochir Rasulsoda nimmt an der Sitzung des Rates der Regierungschefs der GUS-Staaten in } \\
\text { Astana teil. }\end{array}$ \\
\hline 2.11.2018 & $\begin{array}{l}\text { Ein Gericht in Duschanbe verurteilt den Sohn des ehemaligen Energieministers Scherali Gul, Dilowar Gulow, } \\
\text { zu einem Jahr Strafkolonie, nachdem er im Juli } 2018 \text { einen Polizisten bei einer Verkehrskontrolle kranken- } \\
\text { hausreif geschlagen hatte. }\end{array}$ \\
\hline 5.11 .2018 & $\begin{array}{l}\text { In der Nacht schießt der Chef der OMON-Truppen des Autonomen Gebiets Berg-Badachschan (GBAO), } \\
\text { Chursched Muchammadsoda, in Chorog unvermittelt aus einem Auto mit einer Soft Gun auf eine Gruppe } \\
\text { von Jugendlichen. Zwei junge Männer werden verletzt. }\end{array}$ \\
\hline
\end{tabular}




\begin{tabular}{|c|c|}
\hline 6.11 .2018 & $\begin{array}{l}\text { In Chorog protestieren mehrere Dutzend Menschen vor dem Gebäude der Gebietsverwaltung gegen neu in der } \\
\text { Stadt eingerichtete Checkpoints, die Stationierung zusätzlicher Sicherheitskräfte in GBAO und für die Bestra- } \\
\text { fung des Schützen vom Vortag. Der Zugang zu den sozialen Netzwerken, YouTube und einigen Nachrichten- } \\
\text { Websites ist in ganz Tadschikistan blockiert. }\end{array}$ \\
\hline 6.11.2018 & $\begin{array}{l}\text { Der tadschikische Dienst von RFE/RL meldet, dass der von Tadschikistan als IS-Kämpfer via Interpol gesuchte } \\
\text { Murodali Chalimow, der vor zwei Jahren in Kiew verhaftet worden war, nicht ausgeliefert, sondern freigelassen } \\
\text { worden sei. Er soll sich auf dem Territorium der selbsternannten Volksrepublik Donezk befinden. }\end{array}$ \\
\hline 7.11 .2018 & $\begin{array}{l}\text { Außenminister Muchriddin trifft am Rande des CSTO-Treffens in Astana mit seinem kirgisischen Amtskol- } \\
\text { legen Tschingis Aidarbekow zu Gesprächen über die bilateralen Beziehungen zusammen. }\end{array}$ \\
\hline 7.11 .2018 & $\begin{array}{l}\text { In Chudschand (Gebiet Sogd) kommen am späten Abend bei einem Gefangenenaufstand in einem Hochsicher- } \\
\text { heitsgefängnis mehrere Menschen ums Leben, mehreren Insassen soll die Flucht gelungen sein. Ein Gefangener } \\
\text { soll sich die Waffe eines Mitarbeiters angeeignet und die Wachmannschaften beschossen haben. }\end{array}$ \\
\hline 8.11 .2018 & $\begin{array}{l}\text { Generalstaatsanwalt Jusuf Rachmon trifft in Washington mit dem Direktor des Federal Bureau of Investiga- } \\
\text { tion (FBI), Christopher Wray, zu Gesprächen über eine Zusammenarbeit im Kampf gegen transnationale Kri- } \\
\text { minalität sowie internationalen Terrorismus und Extremismus zusammen. }\end{array}$ \\
\hline 8.11 .2018 & $\begin{array}{l}\text { Der IS reklamiert in einer über seine Nachrichtenagentur Amaq verbreiteten Erklärung den Aufstand im Gefäng- } \\
\text { nis von Chudschand für sich, er sei von einem »Soldaten des Kalifats« provoziert worden. }\end{array}$ \\
\hline 8.11 .2018 & $\begin{array}{l}\text { Präsident Rachmon nimmt an der regulären Sitzung des Rates für kollektive Sicherheit der CSTO in Astana } \\
\text { statt, bei der Fragen der Koordinierung der Außenpolitik der Mitgliedsstaaten und eine Verbesserung der } \\
\text { Mechanismen der Zusammenarbeit erörtert werden. }\end{array}$ \\
\hline 9.11 .2018 & $\begin{array}{l}\text { In Duschanbe wird im Beisein von Präsident Rachmon ein Zentrum für den Kampf gegen Terrorismus, Extre- } \\
\text { mismus und Separatismus beim Innenministerium eröffnet. }\end{array}$ \\
\hline 11.11 .2018 & $\begin{array}{l}\text { Der Sprecher der unteren Kammer des Parlamentes, Schukurdschon Suchurow, nimmt in Paris an den Gedenk- } \\
\text { feiern anlässlich des Endes des Ersten Weltkrieges vor } 100 \text { Jahren und dem anschließenden Friedensforum teil. }\end{array}$ \\
\hline 11.11 .1018 & $\begin{array}{l}\text { In seiner Rede anlässlich des Tages der Polizei erklärt Präsident Rachmon, dass der Überfall auf die ausländi- } \\
\text { schen Radtouristen bei Dangara im Juli } 2018 \text { wegen Nachlässigkeit der örtlichen Sicherheitskräfte der Stadt } \\
\text { Nurek und des Bezirks Pjandsch möglich war. }\end{array}$ \\
\hline 12.11 .2018 & $\begin{array}{l}\text { Die russische Nachrichtenagentur RIA Novosti meldet unter Berufung auf eine Quelle im tadschikischen Staat- } \\
\text { lichen Komitee für nationale Sicherheit (GKNB), dass Sicherheitskräfte am 4.11.2018 } 12 \text { IS-Anhänger festge- } \\
\text { nommen hätten, die Terroranschläge auf die 201. russische Militärbasis und die Schule des Verteidigungsmi- } \\
\text { nisteriums der RF in Duschanbe geplant hätten. Ein Sprecher der GKNB dementiert sowohl die Verhaftung } \\
\text { als auch das Bestehen der Gruppe und etwaige Anschlagspläne. }\end{array}$ \\
\hline 13.11 .2018 & $\begin{array}{l}\text { Der Zentralasienexperte von Human Rights Watch, Steve Sverdlov, fordert die tadschikische Regierung zur } \\
\text { transparenten Untersuchung der Vorgänge im Hochsicherheitsgefängnis von Chudschand unter Hinzuziehung } \\
\text { internationaler Spezialisten auf. Bislang ist selbst die Zahl der Todesopfer unklar, oppositionelle Exilmedien } \\
\text { berichten von bis zu } 53 \text { Toten, darunter zwei Gefängniswärter, und } 186 \text { Verletzten. Anlass des Aufstandes soll } \\
\text { die Schikanierung eines Gefangenen durch das Wachpersonal gewesen sein. }\end{array}$ \\
\hline 12.11 .2018 & $\begin{array}{l}\text { Die UN-Frauenrechtskommission fordert die tadschikische Regierung auf, Jungfräulichkeitstests vor der Ehe- } \\
\text { schließung zu verbieten. Sie werden seit durch eine Gesetzesänderung } 2016 \text { ein Gesundheitstest der Heirats- } \\
\text { willigen vor der Eheschließung obligatorisch wurde zunehmend durchgeführt. }\end{array}$ \\
\hline 14.11 .2018 & $\begin{array}{l}\text { Ein Moskauer Gericht verurteilt den Chef des Bestattungsdienstes Ritual, Aleksandr Tschabujew, zu einer Frei- } \\
\text { heitsstrafe von } 11 \text { Jahren und den Anführer der Gruppe "Gesunde Nation«, Aleksandr Botscharnikow, zu zehn } \\
\text { Jahren, wegen der Organisation der Schlägereien auf dem Chowanskij-Friedhof im Frühjahr 2018. Ihr Ziel } \\
\text { bei der Massenschlägerei, an der ca. } 200 \text { Menschen beteiligt waren, sei es gewesen, die tadschikischen Arbeiter } \\
\text { vom Friedhof zu verdrängen. Im Dezember } 2017 \text { waren aus gleichem Anlass bereits fünf tadschikische Staats- } \\
\text { bürger zu jeweils drei Jahren Haft verurteilt worden. }\end{array}$ \\
\hline 15.11 .2018 & $\begin{array}{l}\text { Die ADB bewilligt einen Kredit in Höhe von } 35 \text { Mio. US-Dollar für den Wiederanschluss Tadschikistans an } \\
\text { das zentralasiatische Stromnetz. }\end{array}$ \\
\hline 15.11 .2018 & $\begin{array}{l}\text { Ein Gericht in Duschanbe verurteilt einen Englischlehrer wegen Anwerbung von IS-Kämpfern zu einer 19-jäh- } \\
\text { rigen Freiheitsstrafe. Neun weitere Angeklagte, darunter vier Schüler des Hauptangeklagten, müssen für sechs } \\
\text { Monate bis zu } 19 \text { Jahren hinter Gitter. }\end{array}$ \\
\hline
\end{tabular}




\begin{tabular}{|c|c|}
\hline 15.11.2018 & $\begin{array}{l}\text { Der Leiter der Hochsicherheitsgefängnisses von Chudschand, in dem es am 7.11. zu einem Gefangenenaufstand } \\
\text { gekommen war, wird wegen Fahrlässigkeit, Folter und Verheimlichung des sich anbahnenden Aufstandes } \\
\text { verhaftet. }\end{array}$ \\
\hline 16.11 .2018 & $\begin{array}{l}\text { Präsident Rachmon setzt offiziell den ersten Generator des Wasserkraftwerkes von Rogun in Kraft. An dem fei- } \\
\text { erlichen Ereignis nehmen u. a. der afghanische Vize-Premier Muhammad Khan und der russische stellvertre- } \\
\text { tende Energieminister Andrej Tscheresow sowie der Vize-Präsident der Weltbank, Cyril Muller, teil. }\end{array}$ \\
\hline 17.11.2018 & $\begin{array}{l}\text { Nach Angaben des stellvertretenden Vorsitzende des GKNB, Mansurdschon Umarow, vor dem Parlament, hal- } \\
\text { ten sich derzeit } 1899 \text { tadschikische Kämpfer im Irak und in Syrien auf. Außerdem würden mehrere hundert } \\
\text { Familienangehörige gefallener Kämpfer dort festsitzen. Angaben zur Zahl der Toten macht Umrarow nicht. }\end{array}$ \\
\hline 19.11.2018 & $\begin{array}{l}\text { Tadschikistan stimmt, wie Kasachstan, Kirgistan, Usbekistan, Russland und } 21 \text { weitere Staaten im 3. Komitee } \\
\text { der UN-Vollversammlung gegen einen von der Ukraine initiierten Resolutionsentwurf, der die Annexion der } \\
\text { Krim und Menschenrechtsverletzungen auf der Halbinsel verurteilt. } 67 \text { Staaten stimmen dafür, } 82 \text { enthalten sich. }\end{array}$ \\
\hline 19.11.2018 & $\begin{array}{l}\text { Sieben bei dem Gefangenenaufstand im Hochsicherheitsgefängnis von Chudschand verletzte Wärter werden im } \\
\text { Krankenhaus unter dem Verdacht des Verschweigens der Vorbereitung einer Straftat und Nachlässigkeit verhaftet. }\end{array}$ \\
\hline 19.11.2018 & $\begin{array}{l}\text { Im neuen Bericht der U.S. Commission on International Religious Freedom (USCIRF) für } 2017 \text { wird Tad- } \\
\text { schikistan erneut unter den Ländern, die die religiöse Freiheit verletzen, genannt. }\end{array}$ \\
\hline 20.11.2018 & $\begin{array}{l}\text { Präsident Rachmon empfängt den Sprecher der unteren Kammer des usbekischen Parlamentes, Nurdindschon } \\
\text { Ismoilow, der als erster Inhaber dieses Amtes Tadschikistan besucht, zu Gesprächen über Fragen der bilate- } \\
\text { ralen Beziehungen beider Staaten. Ismoilow führt auch Gespräche mit seinem tadschikischen Amtskollegen } \\
\text { Schukurdschon Suchurow. }\end{array}$ \\
\hline 21.11.2018 & $\begin{array}{l}\text { Das Oberste Gericht verurteilt den Hauptangeklagten im Fall des Angriffs auf die ausländischen Radfahrer, } \\
\text { Abdusamadow, zu einer lebenslänglichen Freiheitsstrafe, zwei weitere Angeklagte müssen für } 16 \text { Jahre hinter } \\
\text { Gitter, die übrigen } 12 \text { für ein bis anderthalb Jahre. }\end{array}$ \\
\hline 21.11.2018 & $\begin{array}{l}\text { Drei tadschikische Staatsbürger, die wegen Teilnahme an Kriegshandlungen im Ausland gesucht wurden, } \\
\text { kehren nach den gesetzlich vorgeschriebenen Gesprächen für freiwillige Rückkehrer ohne Probleme zu ihren } \\
\text { Familien zurück. }\end{array}$ \\
\hline 21.11.2018 & $\begin{array}{l}\text { Der Sekretär des Sicherheitsrates Tadschikistans, Abdurachim Kachcharow, nimmt in Moskau am jährlichen } \\
\text { Treffen der Sekretäre der Sicherheitsräte der GUS-Staaten unter Vorsitz des Vertreters der RF, Nikolaj Patru- } \\
\text { schew, teil, bei dem Fragen der nationalen und internationalen Sicherheit im GUS-Gebiet erörtert werden. }\end{array}$ \\
\hline 22.11 .2018 & $\begin{array}{l}\text { Ein Gericht in Chudschand verurteilt eine Gruppe von acht jungen Männern wegen Mitgliedschaft in der ver- } \\
\text { botenen religiösen Gruppierung der Salafiten zu Freiheitsstrafen von sechs Monaten bis achteinhalb Jahren. }\end{array}$ \\
\hline 23.11.2018 & $\begin{array}{l}\text { Tadschikistan nimmt im neuesten Speedtest Global Index, der die Geschwindigkeit des Internets misst, den } \\
\text { 124. und damit letzten Platz noch hinter Afghanistan und dem Irak ein. }\end{array}$ \\
\hline 23.11.2018 & $\begin{array}{l}\text { In Brüssel findet eine reguläre Sitzung des Rates für die Zusammenarbeit EU-Zentralasien unter Teilnahme } \\
\text { von Außenminister Muchriddin und seinen Amtskollegen aus Kasachstan, Kirgistan, Turkmenistan und Usbe- } \\
\text { kistan sowie der österreichischen Ministerin für Europäische Angelegenheiten, Integration und auswärtige } \\
\text { Angelegenheiten, Karin Kneissl, statt. }\end{array}$ \\
\hline
\end{tabular}

\section{Turkmenistan}

\begin{tabular}{|l|l|}
\hline 22.10.2018 & $\begin{array}{l}\text { Fergananews berichtet, dass die Polizei begonnen habe, Autos auf dem Weg von Aschgabat in die Gebiete } \\
\text { des Landes zu kontrollieren und die Ausfuhr von großen Mengen Lebensmitteln mit Geldstrafen zu belegen. }\end{array}$ \\
\hline 23.10 .2018 & $\begin{array}{l}\text { In Aschgabat findet das dritte mehrtägige Treffen der Vertreter der kaspischen Anrainerstaaten (Turkmenistan, } \\
\text { Russland, Kasachstan, Aserbaidschan, Iran) zur Besprechung des Protokolls zur Bekämpfung der Wilderei statt. }\end{array}$ \\
\hline 26.10 .2018 & $\begin{array}{l}\text { Der turkmenische Dienst von RFE/RL berichtet, dass es auf den Märkten des Gebietes Daschogus so gut } \\
\text { wie kein Mehl mehr gebe. Staatliche Lebensmittelläden würden noch importiertes Mehl verkaufen, aber die } \\
\text { Schlangen seien riesig und es komme aufgrund des Defizits und der langen Wartezeiten häufig zu Konflikten } \\
\text { und Handgreiflichkeiten. }\end{array}$ \\
\hline 29.10 .2018 & $\begin{array}{l}\text { Das Außenministerium empfängt eine deutsche Parlamentariergruppe zum Austausch über die Intensivierung } \\
\text { der interparlamentarischen Beziehungen und die regionale Sicherheitslage. }\end{array}$ \\
\hline
\end{tabular}




\begin{tabular}{|c|c|}
\hline 29.10 .2018 & $\begin{array}{l}\text { Bei einem Treffen der tadschikisch-turkmenischen Regierungskommission für Handel, wirtschaftliche, wissen- } \\
\text { schaftliche und technische Zusammenarbeit in Duschanbe wird die Fertigstellung des Baus der Eisenbahnlinie } \\
\text { Tadschikistan-Afghanistan-Turkmenistan vereinbart. Das Projekt, das } 2013 \text { beschlossen wurde, lag zuletzt } \\
\text { wegen Meinungsverschiedenheiten über die Länge der Streckenabschnitte brach. }\end{array}$ \\
\hline 1.11 .2018 & $\begin{array}{l}\text { Laut dem turkmenischen Dienst von RFE/RL wurden 14.000-15.000 Schüler, Studenten und Angestellte } \\
\text { staatlicher Organisationen gezwungen, an der Vorbereitung der Weltmeisterschaft der Gewichtheber teilzu- } \\
\text { nehmen, die am selben Tag in Aschgabat beginnt. }\end{array}$ \\
\hline 1.11 .2018 & $\begin{array}{l}\text { Der turkmenische Dienst von RFE/RL berichtet, dass die Behörden im Gebiet Daschogus den Bürgern, die } \\
\text { die im vergangenen Monat eingeführten Gebühren für öffentliche Versorgungsleistungen wie Gas, Strom und } \\
\text { Wasser nicht bezahlen, das Gas abdrehen. }\end{array}$ \\
\hline 3.11 .2018 & $\begin{array}{l}\text { In Aschgabat fordern die Behörden alle Einwohner auf, Gaszähler in ihren Wohnungen zu installieren und } \\
\text { überprüfen dies bei Hausbesuchen. Der Preis für einen Zähler inkl. Installation liegt nach offiziellen Angaben } \\
\text { bei } 258 \text { Manat (ca. } 74 \text { US-Dollar). }\end{array}$ \\
\hline 5.11 .2018 & $\begin{array}{l}\text { Der afghanische Finanzminister Mohammad Homayoun Qayumi ist für Gespräche über die Umsetzung } \\
\text { gemeinsamer Infrastrukturprojekte, wie den Bau der TAPI-Pipeline und eines gemeinsamen Eisenbahnkorri- } \\
\text { dors, zu Gast im Außenministerium. }\end{array}$ \\
\hline 6.11 .2018 & $\begin{array}{l}\text { Die offizielle Website des Außenministeriums berichtet, dass Vertreter der OSZE, von UN-Agenturen und der } \\
\text { diplomatischen Vertretungen aus Deutschland, Frankreich, Italien, Großbritannien und der USA Bildungsein- } \\
\text { richtungen in einem Gefängnis im Gebiet Achal besichtigt haben und sich mit Gefangenen unterhalten konnten. }\end{array}$ \\
\hline 10.11 .2018 & $\begin{array}{l}\text { Der türkische Außenminister Mevlüt Çavuşoğlu ist für Gespräche über Handelsbeziehungen und Investitio- } \\
\text { nen mit Außenminister Raschid Meredow in Aschgabat. }\end{array}$ \\
\hline 11.11 .2018 & $\begin{array}{l}\text { Der turkmenische Dienst von RFE/RL berichtet, dass staatliche Bedienstete aus den Bereichen Kultur, Bil- } \\
\text { dung und Öl- und Gasförderung im Gebiet Balkan seit mehreren Wochen kein Gehalt mehr erhalten haben. }\end{array}$ \\
\hline 13.11.2018 & $\begin{array}{l}\text { Der turkmenische Dienst von RFE/RL berichtet, dass im Gebiet Daschogus seit einigen Wochen Kühe und Rin- } \\
\text { der an einer unbekannten Krankheit sterben. Ende Oktober sind im gleichen Gebiet bereits massenweise Kamele } \\
\text { verendet. Wie sich später herausstellte, war die Todesursache jedoch keine Epidemie, sondern Unterernährung. }\end{array}$ \\
\hline 14.11 .2018 & $\begin{array}{l}\text { Laut dem turkmenischen Dienst von RFE/RL können turkmenische Studenten in Russland erneut an Geld- } \\
\text { automaten mit Bankkarten turkmenischer Banken kein Geld abheben. }\end{array}$ \\
\hline 14.11 .2018 & $\begin{array}{l}\text { Präsident Gurbanguly Berdymuchammedow nimmt an der Einweihung des Hauses der turkmenisch-usbeki- } \\
\text { schen Freundschaft im usbekischen Gebiet Urgentsch teil und tauscht sich mit dem usbekischen Außenminis- } \\
\text { ter Abdulasis Kamilow über die bilaterale Zusammenarbeit aus. }\end{array}$ \\
\hline 15.11.2018 & $\begin{array}{l}\text { Präsident Berdymuchammedow empfängt den Präsidenten der Asian Development Bank (ADB), Takehiko } \\
\text { Nakao, zu Gesprächen über die Entwicklung der Partnerschaft zwischen Usbekistan und der ADB. }\end{array}$ \\
\hline 19.11.2018 & $\begin{array}{l}\text { Die Regierung gibt bekannt, dass Saudi-Arabien einen Auftrag in Höhe von } 40 \text { Mio. US-Dollar zur Lieferung } \\
\text { von mehr als } 35.000 \mathrm{~m} \text { Stahlrohren für den Bau des turkmenischen Abschnitts der TAPI-Erdgasleitung erhal- } \\
\text { ten hat. Die Finanzierung erfolgt durch den Saudi Development Fund. Im Februar } 2018 \text { hatte die Regierung } \\
\text { bereits die Fertigstellung des turkmenischen Abschnitts gemeldet. }\end{array}$ \\
\hline 22.11 .2018 & $\begin{array}{l}\text { Präsident Berdymuchammedow empfängt seinen aserbaidschanischen Amtskollegen Ilham Alijew in Asch- } \\
\text { gabat zu Gesprächen, bei denen insgesamt } 20 \text { bilaterale Dokumente über die Zusammenarbeit in den Berei- } \\
\text { chen Handel und Wirtschaft, Verkehr, Transit, Logistik, Bildung, Wissenschaft, Kultur, Investitionsschutz } \\
\text { und Steuern unterzeichnet werden. }\end{array}$ \\
\hline
\end{tabular}

\section{Usbekistan}

\begin{tabular}{|l|l|}
\hline 20.10.2018 & $\begin{array}{l}\text { Präsident Schawkat Mirsijojew und seine Amtskollegen aus Russland und Kasachstan, Wladimir Putin und Nur- } \\
\text { sultan Nasarbajew, treffen im kasachstanischen Saryagasch (Gebiet Turkistan) zu unangekündigten, informel- } \\
\text { len Gesprächen über die trilaterale Zusammenarbeit im ökonomischen und humanitären Bereich zusammen. }\end{array}$ \\
\hline 21.10 .2018 & $\begin{array}{l}\text { Die Arbeitsgruppen der usbekischen und kirgisischen Regierungen zur Regulierung der offenen Grenzfragen } \\
\text { beenden eine weitere Verhandlungsrunde, bei der u. a. die Grenzen der Exklave Schachimardan festgelegt wurden. }\end{array}$ \\
\hline 22.10 .2018 & $\begin{array}{l}\text { In den vergangenen beiden Wochen wurden nach Meldungen usbekischer Medien im Zusammenhang mit Pro- } \\
\text { blemen bei der Baumwollernte zehn Vorsitzende von Städten und Bezirken ausgetauscht, der Gouverneur des } \\
\text { Gebietes Taschkent erhielt einen mündlichen Tadel wegen Nichterfüllung des Ernteplanes. }\end{array}$ \\
\hline
\end{tabular}




\begin{tabular}{|c|c|}
\hline 23.10 .2018 & $\begin{array}{l}\text { Präsident Mirsijojew unterzeichnet eine Verordnung über die Bildung eines Energieministeriums, das für die } \\
\text { Organisation und Regulierung des Kraftstoff- und Energiesektors zuständig sein wird. }\end{array}$ \\
\hline 23.10 .2018 & $\begin{array}{l}\text { Präsident Mirsijojew empfängt US-Handelsminister Wilbur Ross zu Gesprächen über Fragen der Realisierung } \\
\text { verschiedener Programme und Projekte in den Bereichen Handel, Industrie, Landwirtschaft und Transport. }\end{array}$ \\
\hline 25.10 .2018 & $\begin{array}{l}\text { Präsident Mirsijojew unterzeichnet eine Verordnung über erleichterte Regeln zur Gewährung der Staatsbürger- } \\
\text { schaft für langfristig im Ausland lebende Usbeken. Bisher droht dieser Personengruppe bei der Einreise nach } \\
\text { Usbekistan die Verhaftung wegen illegaler Ein- oder Ausreise und bis zu zehn Jahre Gefängnis. }\end{array}$ \\
\hline 27.10 .2018 & $\begin{array}{l}\text { Der Sondergesandte für Afghanistan, Ismatulla Irgaschew, erklärt bei einem Treffen mit dem afghanischen } \\
\text { Berater für nationale Sicherheit, Hamdullah Mohib, dass Usbekistan in den Friedensbemühungen für Afgha- } \\
\text { nistan auch in Kontakt mit den Taliban stehe. }\end{array}$ \\
\hline 28.10 .2018 & $\begin{array}{l}\text { Die russische Komsomolskaja Prawda berichtet, dass ein usbekischer Staatsbürger, der im Donbass auf Seiten } \\
\text { der Separatisten gekämpft hatte und festgenommen worden war, nach dem Einsatz mehrerer russischer Politi- } \\
\text { ker freigelassen wurde, womit ihm auch keine Auslieferung nach Usbekistan mehr droht. }\end{array}$ \\
\hline 29.10 .2018 & $\begin{array}{l}\text { Präsident Mirsijojew entlässt den stellvertretenden Premierminister (mit Zuständigkeit für Land- und Was- } \\
\text { serwirtschaft sowie Lebensmittelproduktion), Zojir Mirsajew, wegen ernster Mängel bei der Organisation der } \\
\text { Aussaat des Wintergetreides. Aus einem Kommentar von Premierminister Abdulla Aripow geht hervor, dass } \\
\text { Anlass der Absetzung im Internet verbreitete Fotos von wegen schlechter Arbeitsergebnisse schikanierten Mit- } \\
\text { arbeitern lokaler Verwaltungen waren. }\end{array}$ \\
\hline 31.10 .2018 & $\begin{array}{l}\text { Im neuesten Doing Business Index } 2019 \text { nimmt Usbekistan mit Rang } 76 \text { (von } 190 \text { Ländern) einen um zwei } \\
\text { Plätze schlechteren Rang als im Vorjahr ein. }\end{array}$ \\
\hline 1.11 .2018 & $\begin{array}{l}\text { Ein Gericht des Gebietes Nawoi verurteilt einen im Sommer } 2018 \text { auf Betreiben des usbekischen Dienstes für } \\
\text { staatliche Sicherheit aus der Türkei ausgelieferten Mann wegen Terrorismus zu } 17 \text { Jahren Lagerhaft. Er soll } \\
\text { von } 1994 \text { bis } 1999 \text { der Bewegung der Dschihadisten angehört haben und hatte seit } 1999 \text { in der Türkei gelebt. }\end{array}$ \\
\hline 1.11 .2018 & $\begin{array}{l}\text { Während der 6. Internationalen Konferenz der Regionalen Antiterrorstruktur (RATS) der SCO in Taschkent } \\
\text { gibt der Direktor des Exekutivkomitees der RATS, Jewgenij Sysojew, bekannt, dass die Sicherheitsdienste der } \\
\text { SCO-Mitgliedsstaaten } 2017 \text { mehr als } 50 \text { Terrorzellen ausgehoben und } 360 \text { Nutzer von terroristischen und reli- } \\
\text { giös-extremistischen Internetgruppen verhaftet haben. }\end{array}$ \\
\hline 1.11 .2018 & $\begin{array}{l}\text { In Taschkent findet das regelmäßige Treffen des Usbekistan - EU Komitees für parlamentarische Zusammen- } \\
\text { arbeit statt, an dem Vertreter des Europäischen Parlamentes und beider Kammern der usbekischen Volksver- } \\
\text { tretung sowie Journalisten teilnehmen. }\end{array}$ \\
\hline 2.11.2018 & $\begin{array}{l}\text { Die Regierung beschließt eine Erhöhung der Preise für Strom (ab 16.11. kostet } 1 \mathrm{kWh} \text { Strom Privatverbrau- } \\
\text { cher 21,4 Sum mehr, am 1.6.2019 folgt eine weitere Erhöhung um } 30 \text { Sum auf } 280 \text { Sum), Gas (am 16.11. und } \\
\text { 1.6.2019 um insgesamt ca. } 50 \text { Sum) und Benzin und Diesel ab 16.11. um 4,5-17\%. }\end{array}$ \\
\hline 2.11 .2018 & $\begin{array}{l}\text { Der Vorsitzende der Liberaldemokratischen Partei Russlands, Wladimir Schirinowskij, besucht mit einer Gruppe } \\
\text { von Duma-Abgeordneten seiner Fraktion Buchara. }\end{array}$ \\
\hline 3.11 .2018 & $\begin{array}{l}\text { Der usbekische Dienst von RFE/RL berichtet, dass am 1.11. die gesamte Auflage der Zeitung Adabijot (Litera- } \\
\text { tur) wegen eines Artikels des Schriftstellers Mamadali Machmudow beschlagnahmt wurde, in dem der ehema- } \\
\text { lige politische Gefangene behauptet, dass der wegen seiner Beteiligung an den Bombenanschlägen von Tasch- } \\
\text { kent } 2009 \text { in absentia verurteilte Muhammad Salih nichts mit diesen Ereignissen zu tun hatte. }\end{array}$ \\
\hline 7.11.2018 & $\begin{array}{l}\text { Der in den Niederlanden ansässige Wohltätigkeitsfonds Friends of Nucus Museum, FoNM, kündigt seine } \\
\text { Auflösung zum } 31.12 .2018 \text { an. Die } 2001 \text { zur Unterstützung des Sawitskij-Museums gegründete Organisation } \\
\text { beklagt das Verschwinden der von ihr gestifteten Audioführer und Bücher. }\end{array}$ \\
\hline 7.11.2018 & $\begin{array}{l}\text { Aus einem Facebook-Post der Direktorin von AI in der Ukraine, Oksana Pokaltschuk, geht hervor, dass die } \\
\text { Generalstaatsanwaltschaft der Ukraine den Antrag Usbekistans auf Ausweisung des Journalisten Nasrullo } \\
\text { Achundschonow abgelehnt hat. }\end{array}$ \\
\hline 8.11 .2018 & $\begin{array}{l}\text { Verteidigungsminister Abdusalom Asisow empfängt eine US-amerikanische Militärdelegation unter Führung } \\
\text { von Deputy Assistant Secretary of Defense for Afghanistan, Pakistan and Central Asia, Colin Jackson, zu } \\
\text { Gesprächen über Stand und Perspektiven der militärischen Zusammenarbeit. }\end{array}$ \\
\hline 8.11 .2018 & $\begin{array}{l}\text { Außenminister Abdulasis Kamilow trifft während seines Deutschlandbesuches u. a. mit Bundesaußenminis- } \\
\text { ter Heiko Maas zu Gesprächen über Stand und Perspektiven der bilateralen Zusammenarbeit, die Situation in } \\
\text { Afghanistan und die Möglichkeiten einer Kooperation Usbekistans mit der EU zusammen. }\end{array}$ \\
\hline
\end{tabular}




\begin{tabular}{|c|c|}
\hline 8.11.2018 & $\begin{array}{l}\text { Der Sonderbeauftragte für Afghanistan, Irgaschew, nimmt an der internationalen Konferenz »Joint Vision for } \\
\text { Afghanistan« im Europäischen Parlament in Brüssel teil. }\end{array}$ \\
\hline 9.11 .2018 & $\begin{array}{l}\text { Der Rechtsanwalt des bekanntesten usbekischen Oppositionellen und Vorsitzenden der Demokratischen Par- } \\
\text { tei Erk, Muhammad Solih, teilt mit, dass sein Mandant nun eine offizielle Bestätigung von Interpol über seine } \\
\text { Streichung von der Liste der international gesuchten Personen habe. Solih, der in der Türkei lebt, wurde auf } \\
\text { usbekisches Betreiben seit } 1999 \text { international gesucht und wurde mehrfach bei Auslandsreisen festgenommen, } \\
\text { aber immer wegen des politischen Hintergrundes des Suchbefehls wieder freigelassen. }\end{array}$ \\
\hline 9.11 .2018 & $\begin{array}{l}\text { Ein Gericht im kasachstanischen Aktobe verurteilt die drei Fahrer des Autobusses, der am 18.1.2018 auf der } \\
\text { Überlandstraße Samara-Schymkent ausgebrannt war, zu fünf Jahren Freiheitsentzug. Der Gründer der Firma } \\
\text { und der zuständige Automechaniker müssen für dreieinhalb Jahre hinter Gitter. Bei dem Feuer waren } 52 \text { usbe- } \\
\text { kische Arbeitsmigranten zu Tode gekommen. }\end{array}$ \\
\hline 11.11 .2018 & $\begin{array}{l}\text { Der Vorsitzende des Senats, Nigmatilla Juldaschew, nimmt an den Feierlichkeiten anlässlich des 100. Jahres- } \\
\text { tages des Endes des Zweiten Weltkrieges in Paris teil. }\end{array}$ \\
\hline 12.11 .2018 & $\begin{array}{l}\text { Usbekistan unterzeichnet als einziger zentralasiatischer Staat die vom französischen Präsidenten Emmanuel } \\
\text { Macron auf dem UNESCO Forum zur Regulierung des Internets vorgeschlagene Deklaration Paris Call for } \\
\text { Trust and Security in Cyberspace. }\end{array}$ \\
\hline 13.11.2018 & $\begin{array}{l}\text { Der usbekische Dienst von RFE/RL berichtet, dass dem im schweizerischen Exil lebenden Politemigranten } \\
\text { Safar Bekschan bereits am 17.3.2017 ohne Angabe von Gründen die usbekische Staatsbürgerschaft aberkannt } \\
\text { wurde. Er wurde darüber aber erst jetzt durch einen Brief des usbekischen Konsulats in Berlin informiert. }\end{array}$ \\
\hline 16.11.2018 & $\begin{array}{l}\text { Die nicht registrierte oppositionelle Demokratische Volksbewegung Birdomlik konnte nach Angaben ihres } \\
\text { Vorsitzenden Bachodir Hon Turkiston, alias Bachodir Chorijew, ihren für den 15./16.11.2018 im kasachstani- } \\
\text { schen Schymkent geplanten Kongress nicht abhalten, weil viele potentielle Teilnehmer nicht über die Grenze } \\
\text { gelassen bzw. des Landes verwiesen wurden. }\end{array}$ \\
\hline 16.11.2018 & $\begin{array}{l}\text { er Sprecher der unteren Kammer des Parlamentes, Nuridschon Ismailow, wird während seines Arbeitsbesu- } \\
\text { les in Bischkek vom kirgisischen Präsidenten Sooronbaj Dscheenbekow zu Gesprächen empfangen. }\end{array}$ \\
\hline 17.11.2018 & $\begin{array}{l}\text { Human Rights Watch (HRW) fordert in einer auf seiner Website veröffentlichten Erklärung die usbekische } \\
\text { Führung auf, weitere politische Gefangene freizulassen und die bereits aus der Haft entlassenen zu rehabilitie- } \\
\text { ren. Nach Angaben der Menschenrechtsorganisation wurden seit dem Amtsantritt Präsident Mirsijojews mehr } \\
\text { als } 35 \text { aus politischen Motiven Verurteilte frei gelassen, es befinden sich aber noch Tausende wegen zweifel- } \\
\text { hafter Motive hinter Gittern. }\end{array}$ \\
\hline 19.11 .2018 & $\begin{array}{l}\text { Usbekistan stimmt, wie Kasachstan, Kirgistan, Tadschikistan, Russland und } 21 \text { weitere Staaten im 3. Komitee } \\
\text { der UN-Vollversammlung gegen einen von der Ukraine initiierten Resolutionsentwurf, der die Annexion der } \\
\text { Krim und Menschenrechtsverletzungen auf der Halbinsel verurteilt. } 67 \text { Länder stimmen dafür, } 82 \text { enthalten sich. }\end{array}$ \\
\hline 20.11.2018 & $\begin{array}{l}\text { Chafisullo Nasyrow, einer der ersten Führer der Hisb ut-Tahrir, gibt in einem dem usbekischen Dienst von } \\
\text { RFE/RL zugespielten Video seinen Austritt aus der verbotenen religiösen Gruppe bekannt. }\end{array}$ \\
\hline 20.11.2018 & $\begin{array}{l}\text { Der Sprecher der unteren Kammer des usbekischen Parlamentes, Ismoilow, wird in Duschanbe vom tadschi- } \\
\text { kischen Präsidenten Emomali Rachmon und seinem Amtskollegen Schukurdschon Suchurow zu Gesprächen } \\
\text { über Fragen der bilateralen Beziehungen beider Staaten empfangen. Dies ist der erste offizielle Tadschikistan- } \\
\text { Besuch eines Sprechers des usbekischen Parlamentes. }\end{array}$ \\
\hline 21.11.2018 & $\begin{array}{l}\text { Nach Angaben der International Labour Organisation (ILO) arbeiteten während der diesjährigen Baumwoll- } \\
\text { ernte } 93 \% \text { der Pflücker auf freiwilliger Basis, eine Verbesserung gegenüber dem Vorjahr um } 6 \% \text {. Das Minis- } \\
\text { terium für Beschäftigung und Arbeitsbeziehungen teilt mit, dass mehr als } 200 \text { Führungspersonen wegen Aus- } \\
\text { übung von Zwang während der Baumwollernte zur Verantwortung gezogen würden. }\end{array}$ \\
\hline 21.11.2018 & $\begin{array}{l}\text { Präsident Mirsijojew empfängt den Generaldirektor des Büros der UN in Genf, Michael Møller, zu Gesprä- } \\
\text { chen zu Stand und Perspektiven der Zusammenarbeit. }\end{array}$ \\
\hline 21.11.2018 & $\begin{array}{l}\text { Außenminister Kamilow wird in Brüssel von Neven Mimica, EU-Kommissar für Entwicklungszusammenar- } \\
\text { beit, zu Gesprächen über Stand und Perspektiven der Kooperation empfangen. Gleichzeitig wird bekannt, dass } \\
\text { die EU Usbekistan } 25 \text { Mio. Euro zur Unterstützung der ländlichen Bevölkerung zur Verfügung stellt, das Jus- } \\
\text { tizministerium erhält einen } 10 \text { Mio. Euro Kredit. }\end{array}$ \\
\hline 21.11.2018 & $\begin{array}{l}\text { Der Vorsitzende des Senats, Juldaschew, empfängt eine Delegation der Parlamentarischen Versammlung der } \\
\text { OSZE unter Leitung ihres Vorsitzenden George Tsereteli zu Gesprächen über Prioritäten der Zusammenar- } \\
\text { beit beider Volksvertretungen. }\end{array}$ \\
\hline
\end{tabular}




\begin{tabular}{|l|l|}
\hline 22.11.2018 & $\begin{array}{l}\text { Außenminister Kamilow trifft in Brüssel mit der österreichischen Ministerin für Europäische Angelegenhei- } \\
\text { ten, Integration und auswärtige Angelegenheiten, Karin Kneissl, zu Gesprächen über die Beziehungen Usbe- } \\
\text { kistan-EU zusammen. }\end{array}$ \\
\hline 23.11.2018 & $\begin{array}{l}\text { Außenminister Kamilow und die Hohe Vertreterin der EU für Außen- und Sicherheitspolitik, Federica Mog- } \\
\text { herini, beginnen die offiziellen Verhandlungen über ein Erweitertes Partnerschafts- und Kooperationsabkom- } \\
\text { men (EPCA). Am gleichen Tag findet in Brüssel auch die reguläre Sitzung des Rates für die Zusammenarbeit } \\
\text { EU-Zentralasien unter Teilnahme der Außenminister aller zentralasiatischen Staaten statt. }\end{array}$ \\
\hline
\end{tabular}

Sie können die gesamte Chronik seit 2008 auch auf <http://www.laender-analysen.de/zentralasien/> unter dem Link »Chronik«lesen.

\section{Herausgeber:}

Zentrum für Osteuropa- und internationale Studien (ZOiS) gGmbH Deutsche Gesellschaft für Osteuropakunde e.V.

Deutsches Polen-Institut

Forschungsstelle Osteuropa an der Universität Bremen

Leibniz-Institut für Agrarentwicklung in Transformationsökonomien

Leibniz-Institut für Ost- und Südosteuropaforschung

Redaktion:

Dr. Beate Eschment (verantwortlich)

Redaktionsassistenz: Ann-Sophie Gast

Satz: Matthias Neumann

Wissenschaftlicher Beirat:

Dr. Luca Anceschi, University of Glasgow

Prof. Dr. Martin Petrick, Leibniz-Institut für Agrarentwicklung in Transformationsökonomien, Halle

Die Meinungen, die in den Zentralasien-Analysen geäußert werden, geben ausschließlich die Auffassung der Autoren wieder. Abdruck und sonstige publizistische Nutzung sind nach Rücksprache mit der Redaktion gestattet.

Zentralasien-Analysen-Layout: Matthias Neumann, nach einen Konzept von Cengiz Kibaroglu, mit einer Grafik von Sebastian Klüsener

Alle Ausgaben der Zentralasien-Analysen sind mit Themen- und Autorenindex archiviert unter www.laender-analysen.de

ISSN 1866-2110 @ 2018 by Zentrum für Osteuropa- und internationale Studien (ZOiS) gGmbH, Deutsche Gesellschaft für Osteuropakunde e.V., Deutsches Polen-Institut, Forschungsstelle Osteuropa an der Universität Bremen, Leibniz-Institut für Agrarentwicklung in Transformationsökonomien, Leibniz-Institut für Ost- und Südosteuropaforschung

Zentrum für Osteuropa- und internationale Studien (Z0is) gGmbH • Mohrenstraße 60 • 10117 Berlin • Telefon: + 49 (30) 2005949-20

e-mail: beate.eschment@zois-berlin.de•Internet-Adresse: www.laender-analysen.de/zentralasien 


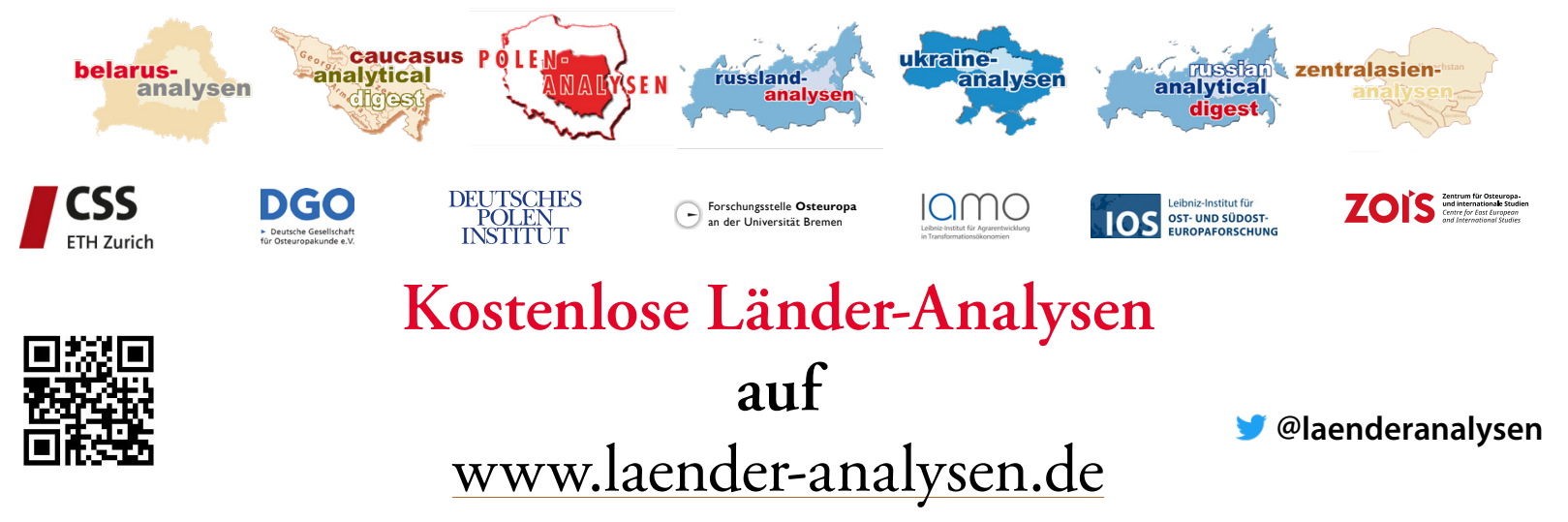

Die Länder-Analysen bieten regelmäßig im kostenlosen Abonnement kompetente Einschätzungen aktueller politischer, wirtschaftlicher, sozialer und kultureller Entwicklungen in Ostmitteleuropa und der GUS. Alle Länder-Analysen verstehen sich als Teil eines gemeinsamen Projektes, das der wissenschaftlich fundierten, allgemeinverständlich formulierten Analyse der Entwicklungen im östlichen Europa, der Offenheit für verschiedene inhaltliche Positionen und der kostenlosen und nicht-kommerziellen Information einer breit verstandenen interessierten Öffentlichkeit verpflichtet ist. Autor/innen sind internationale Fachwissenschaftler/innen und Expert/innen. Die Redaktionen der Länder-Analysen bestehen aus Wissenschaftler/innen mit langjähriger Forschungserfahrung.

Die deutschsprachigen Länder-Analysen werden gemeinsam von der Forschungsstelle Osteuropa an der Universität Bremen, dem Zentrum für Osteuropa- und internationale Studien, der Deutschen Gesellschaft für Osteuropakunde, dem Deutschen Polen-Institut, dem Leibniz-Institut für Agrarentwicklung in Transformationsökonomien und dem Leibniz-Institut für Ost- und Südosteuropaforschung herausgegeben. Die englischsprachigen Länder-Analysen erscheinen in Kooperation der Forschungsstelle Osteuropa mit dem Center for Security Studies (CSS) der ETH Zürich.

Die Länder-Analysen bieten regelmäßig Kurzanalysen zu aktuellen Themen, ergänzt um Grafiken und Tabellen sowie Dokumentationen. Zusätzlich gibt es eine Chronik aktueller Ereignisse. Alle Länder-Analysen sind auch mit Archiv und Indizes online verfügbar unter $<$ www.laender-analysen.de $>$.

\section{Belarus-Analysen}

Erscheinungsweise: zweimonatlich

Abonnement unter: <http://www.laender-analysen.de/belarus/>

\section{Caucasus Analytical Digest}

In englischer Sprache. Erscheinungsweise: monatlich

Abonnement unter: <http://www.css.ethz.ch/en/publications/cad.html>

\section{Polen-Analysen}

Erscheinungsweise: zweimal monatlich

Abonnement unter: <http://www.deutsches-polen-institut.de/newsletter/polen-analysen/>

Auch als App für Android ${ }^{\mathrm{TM}}$ (ab Januar 2016) kostenlos auf Google Play ${ }^{\mathrm{TM}}$.

\section{Russland-Analysen}

Erscheinungsweise: zweiwöchentlich

Abonnement unter: <http://www.laender-analysen.de/russland/>

Auch als App für Android (ab Januar 2016) kostenlos auf Google Play.

\section{Russian Analytical Digest}

In englischer Sprache. Erscheinungsweise: zweimal monatlich

Abonnement unter: $<$ http://www.css.ethz.ch/en/publications/rad.html>

\section{Ukraine-Analysen}

Erscheinungsweise: zweimal monatlich

Abonnement unter: <http://www.laender-analysen.de/ukraine/>

Auch als App für Android (ab Januar 2016) kostenlos auf Google Play.

\section{Zentralasien-Analysen}

Erscheinungsweise: monatlich

Abonnement unter: <http://www.laender-analysen.de/zentralasien/>

Auch als App für Android (ab Januar 2016) kostenlos auf Google Play.

Google Play, das Google Play-Logo und Android sind Marken von Google Inc. TWITTER, TWEET, RETWEET und das Twitter Logo sind eingetragene Markenzeichen von Twitter, Google Play, das Google Play-Logo und Android sind Marken von Google Inc. TWITTER, TWEET, RETWEET und das Twitter Logo sind eingetragene Markenzeichen von Twitter,
Inc. oder angeschlossenen Unternehmen. Der Android-Roboter wird auf der Grundlage von einem Werk wiedergegeben oder modifiziert, das von Google erstellt und geteilt und gemäß den Bedingungen der Creative Commons 3.0 Attribution-Lizenz verwendet wird. 